\title{
Design and analysis of Lacl-repressed promoters and DNA-looping in a cyanobacterium
}

Daniel Camsund ${ }^{1}$, Thorsten Heidorn ${ }^{2}$ and Peter Lindblad ${ }^{1 *}$

\begin{abstract}
Background: Cyanobacteria are solar-powered prokaryotes useful for sustainable production of valuable molecules, but orthogonal and regulated promoters are lacking. The Lac repressor (Lacl) from Escherichia coli is a well-studied transcription factor that is orthogonal to cyanobacteria and represses transcription by binding a primary lac operator (lacO), blocking RNA-polymerase. Repression can be enhanced through DNA-looping, when a Lacl-tetramer binds two spatially separated lacO and loops the DNA. Ptrc is a commonly used Lacl-repressed promoter that is inefficiently repressed in the cyanobacterium Synechocystis PCC 6803. Ptrc2O, a version of Ptrc with two lacO, is more efficiently repressed, indicating DNA-looping. To investigate the inefficient repression of Ptrc and cyanobacterial DNA-looping, we designed a Ptrc-derived promoter library consisting of single lacO promoters, including a version of Ptrc with a stronger lacO (Ptrc1O-proximal), and dual lacO promoters with varying inter-lacO distances (the Ptrc2O-library).

Results: We first characterized artificial constitutive promoters and used one for engineering a Lacl-expressing strain of Synechocystis. Using this strain, we observed that Ptrc1O-proximal is similar to Ptrc in being inefficiently repressed. Further, the Ptrc2O-library displays a periodic repression pattern that remains for both non- and induced conditions and decreases with longer inter-lacO distances, in both E. coli and Synechocystis. Repression of Ptrc2O-library promoters with operators out of phase is less efficient in Synechocystis than in E. coli, whereas repression of promoters with lacO in phase is efficient even under induced conditions in Synechocystis. Two well-repressed Ptrc2O promoters were highly active when tested in absence of Lacl in Synechocystis.

Conclusions: The artificial constitutive promoters herein characterized can be utilized for expression in cyanobacteria, as demonstrated for Lacl. The inefficient repression of Ptrc and Ptrc1O-proximal in Synechocystis, as compared to E. coli, may be due to insufficient Lacl expression, or differences in RNAP subunits. DNA-looping works as a transcriptional regulation mechanism similarly as in E. coli. DNA-looping contributes strongly to Ptrc2O-library repression in Synechocystis, even though they contain the weakly-repressed primary lacO of Ptrc1O-proximal and relatively low levels of Lacl/cell. Hence, Synechocystis RNAP may be more sensitive to DNA-looping than E. coli RNAP, and/or the chromatin torsion resistance could be lower. Two strong and highly repressed Ptrc2O promoters could be used without induction, or together with an unstable Lacl.

Keywords: Cyanobacteria, Lac repressor, Synthetic biology, Transcriptional systems, Ptrc, Synechocystis, Lacl, Metabolic engineering tools, Transcriptional regulation
\end{abstract}

\footnotetext{
* Correspondence: peter.lindblad@kemi.uu.se

'Microbial Chemistry, Department of Chemistry-Ångström Laboratory, Science for Life Laboratory, Uppsala University, P.O. Box 523, SE-75120 Uppsala, Sweden

Full list of author information is available at the end of the article
} 


\section{Background}

Cyanobacteria, solar-powered prokaryotes that fix $\mathrm{CO}_{2}$ from air during photosynthesis, can be engineered to produce a variety of valuable molecules and fuels sustainably $[1,2]$, and hence present a literally green alternative to the use of fossil fuels. The metabolic engineering efforts of even common biotechnological chassis such as Escherichia coli (E. coli), has illustrated the need for synthetic biology to provide new tools, e.g. minimal chassis, vectors and genetic control systems [3]. This is all the more true for cyanobacteria, and even though tools are available for cyanobacterial synthetic biology, more tools, especially promoters, are needed [4,5]. Further, cellular context effects, cross-talk and interactions among genetic components and control systems are some of many often unpredictable causes for failure in engineered biological systems [6]. Orthogonal biological systems, which display minimal cross-talk because their components are decoupled from each other and preferably from the host, could help mitigate these problems [7]. However, most promoters previously used for engineering cyanobacteria are native to the chassis in question [8], so there is a specific lack of orthogonal promoters, constitutive as well as regulated.

The Lac repressor (LacI) from E. coli is a well-studied tetrameric (a dimer of dimers) transcription factor [9] orthogonal to cyanobacteria that represents a model system and, in combination with an optimized promoter a tool, for engineering synthetic cyanobacterial transcriptional systems. In wild-type $E$. coli, LacI represses transcription of the lacZYA operon mainly by binding its primary lac operator $(\mathrm{lacO} 1)$ that is located downstream of and overlapping with $1 \mathrm{bp}$ the transcription start site (TSS) of the promoter [10]. Repression can be relieved by induction using lactose or its stable analog isopropyl $\beta$-D-1-thiogalactopyranoside (IPTG), which reduces the affinity of LacI towards its operator approximately 1000-fold [11]. The repression of the E. coli lac operon also depends on the auxiliary operators to which LacI binds with differing affinity. Of the three wild-type operators (the primary lacO1 and the auxiliary operators $\mathrm{O} 2$ and $\mathrm{O} 3$ ) that display an approximate dyad symmetry and an artificial perfectly symmetric lacO (lacOsym) [12], lacOsym was found to bind LacI with the greatest affinity, with the binding affinities ordered as lacOsym $>$ lacO $1>\mathrm{lacO} 2>$ lacO3 [13]. Early on, it was found that the auxiliary lac operators, which are spatially separated from the primary lacO1, improve repression through a DNA-looping mechanism that increases the effective local concentration of LacI at the primary repression site [14]. A LacI tetramer bound to separately located lacO on the same side of the DNA-helix would be able to bend or loop the DNA (Figure 1). Vice versa, two lacO on different sides of the helix would not be able to simultaneously bind LacI by simply looping the DNA, making the formation of LacI-

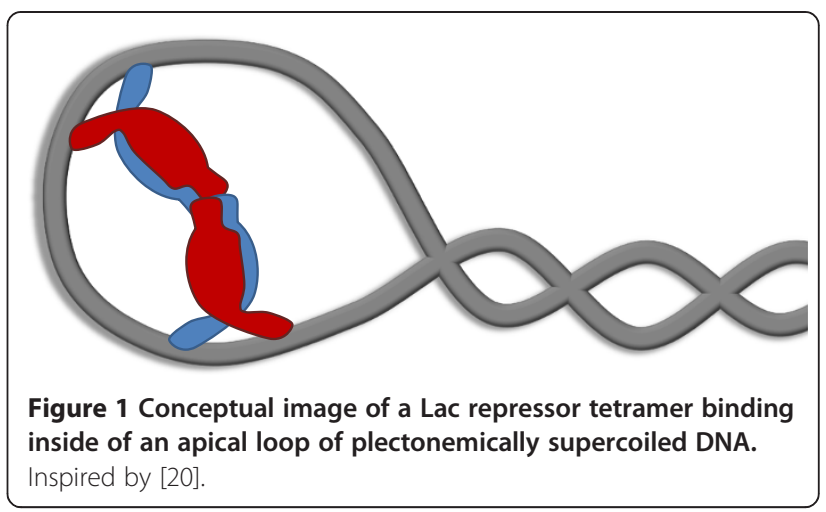

DNA-loops in geometrically unfavorable configurations more difficult. The DNA-looping model of cooperative tetrameric LacI-repression was confirmed in vitro [15], and in vivo [16]. Further, these and subsequent studies showed that DNA-loop-dependent repression varies periodically as a function of the distance between the two operators, with a period of about $11 \mathrm{bp}$ or the approximate numbers of bp per helical turn of supercoiled chromatin DNA in vivo [17-22]. However, it should be noted that the helical repeat of in vivo chromatin DNA (referred to as DNA) will depend on the associated protein factors [20]. In a comprehensive in vivo DNA-looping study using a fixed primary lacO1 for repression of a lacUV5 promoter, the upstream position of an auxiliary lacOsym was varied by varying the length of a spacer between the -35 box and the operator, effectively varying the inter-lacO distance from 57.5 to about $1500 \mathrm{bp}$ [23]. While confirming previous results regarding DNA-loop mediated repression, this study also found that repression is phasedependent from the shortest inter-lacO distance tested up to approximately $200 \mathrm{bp}$, that repression is more effective for shorter distances, and that phasing was not observed for distances above $400 \mathrm{bp}$. The strong repression phasedependency for short inter-lacO distances and the lack of phase-dependency for longer distances was explained by the strong torsion resistance of short segments of DNA, making pure looping more energetically favorable than twisting, and the lower torsion resistance of longer stretches of DNA, removing the barrier for twisted loop formation.

We previously introduced and characterized two LacIregulated promoters, Ptrc [24] and Ptrc2O, in the unicellular model cyanobacterium Synechocystis PCC 6803 (Synechocystis) [25]. Both promoters contain the same Ptrc core promoter, a chimera of the E. coli trp operon and lacUV5 promoters, including the proximal lacO1 from the lac operon, and proved to be highly active in Synechocystis in the absence of LacI. This was expected since Ptrc contains the optimally spaced $E$. coli consensus -35 and -10 elements, which are similar to the consensus -35 and -10 boxes of the cyanobacterial group 1 promoters that are 
recognized by the primary sigma factor under normal growth conditions [26]. However, we observed that Ptrc is less efficiently repressed by heterologously expressed LacI in Synechocystis than in an E. coli strain over-expressing LacI. Further, Ptrc2O, a modified Ptrc with an additional lacOsym inserted further upstream of the -35 element to enable DNA-loop-enhanced repression, was more efficiently repressed in Synechocystis, but could not be efficiently induced [25]. The efficient repression of $\mathrm{Ptrc2O}$, even under full IPTG induction, implies that insufficient levels of heterologously expressed LacI was not the reason for the inefficient repression of Ptrc in Synechocystis. In contrast to these results, Ptrc in combination with the $\operatorname{lacl}^{q}$ gene has proven to be both well-repressed and inducible in the cyanobacterium Synechococcus elongatus PCC 7942 [27]. However, when a recent study used a similar Ptrc and lacl $^{q}$ setup in Synechocystis, no or very little repression of Ptrc could be detected, whereas the contrary was true in E. coli [28], in line with our previous results [25]. Further, Ptac [29], which differs from Ptrc only in that the spacer between the -35 and -10 boxes is $16 \mathrm{bp}$ instead of $17 \mathrm{bp}$ [24], was only partially repressed by LacI when tested in the cyanobacterium Nostoc PCC 7120 [30]. The difference in repression of Ptrc between E. coli and Synechocystis could be due to differences in the cyanobacterial RNA polymerase (RNAP) complex [31,32] and in the interactions between RNAP, promoter and LacI, or simply because of differences in the amount of LacI per cell. Further, Ptrc2O could be efficiently repressed in Synechocystis, implying that DNA-looping through the LacI-tetramer may work in a similar way as in E. coli. While DNA curvature has been previously recognized to affect gene expression in cyanobacteria [33], as far as we are aware, gene regulation through DNA-looping has not previously been experimentally studied in cyanobacteria.

In the present study, we characterized minimal artificial promoters that can be utilized for stable constitutive expression in cyanobacteria, as demonstrated by using one of them to engineer a LacI-expressing strain of Synechocystis. Using this strain, we characterized a version of Ptrc with a stronger lacO, Ptrc1O-proximal, which similarly to Ptrc display limited repression as compared to its more efficient repression in E. coli. Possible reasons for this were found in the differences between $E$. coli and cyanobacterial RNA polymerases, or in the lower number of LacI/cell in the LacI-expressing strain of Synechocystis as compared with the LacI over-expressing strains of $E$. coli herein tested. Further, we characterized the Ptrc2O-library in both E. coli and Synechocystis and observe several similarities and differences between the two species. While the system displays a similar periodic repression pattern in both, illustrating the presence of DNA-looping in cyanobacteria, differences are found. These differences in repression and induction are attributed to the differing repression efficiency of the proximal lac operator of Ptrc1O-proximal, and potential differences between the RNAP sensitivity to DNA-looping and/or the in vivo chromatin DNA torsion-resistance between the two species. These findings will aid in the understanding of native cyanobacterial transcriptional systems and have design implications for engineering synthetic regulated promoters.

\section{Results \\ Characterization of constitutive promoters in Synechocystis}

To investigate the activity of minimal and constitutive orthogonal promoters in the unicellular cyanobacterium Synechocystis PCC 6803 (Synechocystis), eight members from an artificial promoter library spanning a wide range of activities in Escherichia coli were selected for testing using a pPMQAK1-carried [25] RBS* [4] EYFP fluorescent protein reporter construct. The artificial BioBrick promoters BBa_J23100, BBa_J23101, BBa_J23102, BBa_J23105, BBa_J23106, BBa_J23109, BBa_J23113 and BBa_J23114 [34] were compared to three promoters native to Synechocystis; the nitrate reductase promoter PnirA, the plastocyanin promoter PpetE and the RNase P subunit B promoter PrnpB, which have been previously used for engineered expression, using the same reporter construct. The constitutive artificial promoters were found to span a wide range of activities in Synechocystis, encompassing and expanding the range covered by the three native promoters (Figure 2).

\section{Construction of a Lacl-expressing and a Chloramphenicol resistance-only strain of Synechocystis}

In our previous study [25] we found that LacI expressed from pPMQAK1 using PrnpB and the BBa_B0034 [34] ribosome binding site was sufficient for repression of the original Ptrc2O promoter. For constructing a strain of Synechocystis expressing similar levels of LacI from the chromosome instead of from the pPMQAK1 vector, we selected the minimal, constitutive and orthogonal BBa_J23114 promoter (weaker than the PrnpB promoter, as characterized in this study) in combination with the RBS* ribosome binding site that was found to be stronger than BBa_B0034 in Synechocystis [4]. Since the copy number of pPMQAK1 is expected to be similar to the copy number of the Synechocystis chromosome [25] or lower, taking newer data measuring the copy number of the chromosome to about 40 to 220 per cell depending on strain and growth phase into account [35], and assuming that the promoter activities are similar on the PPMQAK1 plasmid and the chromosome, we expected levels of LacI expression to be similar or higher than in our previous study. The LacI-expression cassette, consisting of a forward terminator blocking potentially incoming RNA polymerases from the native part of the chromosome, the 


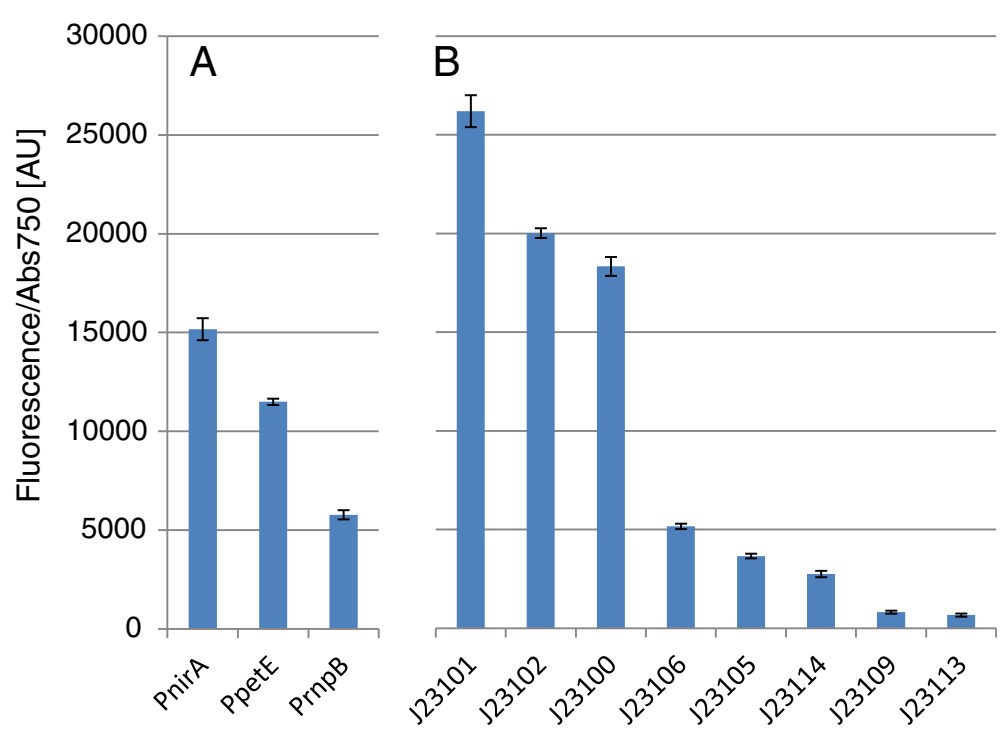

Figure 2 Promoter activities per cell characterized using an EYFP reporter construct carried on PPMQAK1 in Synechocystis PCC 6803. A. Native promoters from Synechocystis included for comparison. PnirA is the nitrate reductase promoter, PpetE is the plastocyanin promoter and PrnpB is the RNase P subunit B promoter. B. Minimal constitutive promoters from an Escherichia coli synthetic promoter library. The J23\#\#\# promoters correspond to the BBa_J23\#\#\# BioBrick parts obtained from the iGEM Registry [34]. Promoter activities per cell are given in fluorescence/absorbance at $750 \mathrm{~nm}$ in arbitrary units [AU]. Error bars indicate standard error of the mean $(n=6)$.

BBa_J23114, RBS* and lacI CDS expression unit, and another forward terminator to stop BBa_J23114-promoted RNAP from reading out into the subsequent chloramphenicol resistance cassette, was inserted into a previously used neutral site of the chromosome using homologous recombination. The obtained LacI-enhanced strain of Synechocystis was confirmed to be fully segregated using PCR with primers flanking the insertion site, and further the areas around the insertion sites were sequence-verified using the same primers. LacI expression was confirmed through western blotting using LacI antibodies (data not shown) and confirmed functional through LacI repression and IPTG induction assays (see following results). Further, as known numbers of cells were used for the western blot of the LacI-enhanced strain of Synechocystis and the two E. coli strains used herein, NEB $5 \alpha$ and DH5 $\alpha \mathrm{Z} 1$, we were able to quantify the relative levels of LacI/cell among the three strains. NEB5 $\alpha$ has about $5 \mathrm{X}$ as many LacI/cell as compared to the LacI-enhanced strain of Synechocystis, and DH5 $\alpha$ Z1 has about $16 \mathrm{X}$ as many LacI/cell. Using a previously published value for the number of LacI tetramers/cell in DH5 $\mathrm{ZZ1}, 3000 /$ cell [36], as a standard, we estimated the number of LacI tetramers/cell in the LacI-enhanced strain of Synechocystis to be 190, and the number of LacI/cell in NEB5 $\alpha$ to be about 880 .

Finally, to enable the characterization of promoters in a control strain grown under the same conditions as the LacI-enhanced strain of Synechocystis but without LacI, we constructed an engineered strain with the chloramphenicol resistance-only inserted into the neutral site.
This chloramphenicol resistance-only strain of Synechocystis was constructed and confirmed in the same way as the LacI-enhanced strain.

\section{Construction and characterization of the Ptrc-derived promoter library in E. coli}

To investigate the limited repression of Ptrc, the limited induction of Ptrc2O that we previously encountered in Synechocystis [25], and the characteristics of LacImediated DNA-looping in cyanobacteria, we designed and constructed a series of Ptrc-derived promoters. This collection consists of control promoters (Figure 3A) and the Ptrc2O-library itself (Figure $3 \mathrm{~B}$ ). Among the control promoters BBa_J23101 serves as a constitutive control and reference. PA1lacO-1 [36] is a strongly-repressed yet highly inducible promoter in $E$. coli that was recently found to be both repressed and inducible in Synechocystis [28]. The original Ptrc2O promoter works as a reference to our previous experiment [25] and Ptrc1O-distal as a Ptrc-derived promoter with the symmetric lac operator (lacOsym) inserted immediately upstream of the -35 element. Ptrc1O-core with a slightly truncated lacOsym inserted in the core of the promoter serves as a control for PAllacO-1 that also contains a core-located lacO. Ptrc1O-proximal is identical to the original Ptrc [24] that we previously characterized [25] except for that we switched the proximal lacO1 with lacOsym. If the low repressibility of Ptrc that we previously observed in Synechocystis is due to lack of LacI binding lacO1, we should observe an increase in repression by switching lacO1 for 


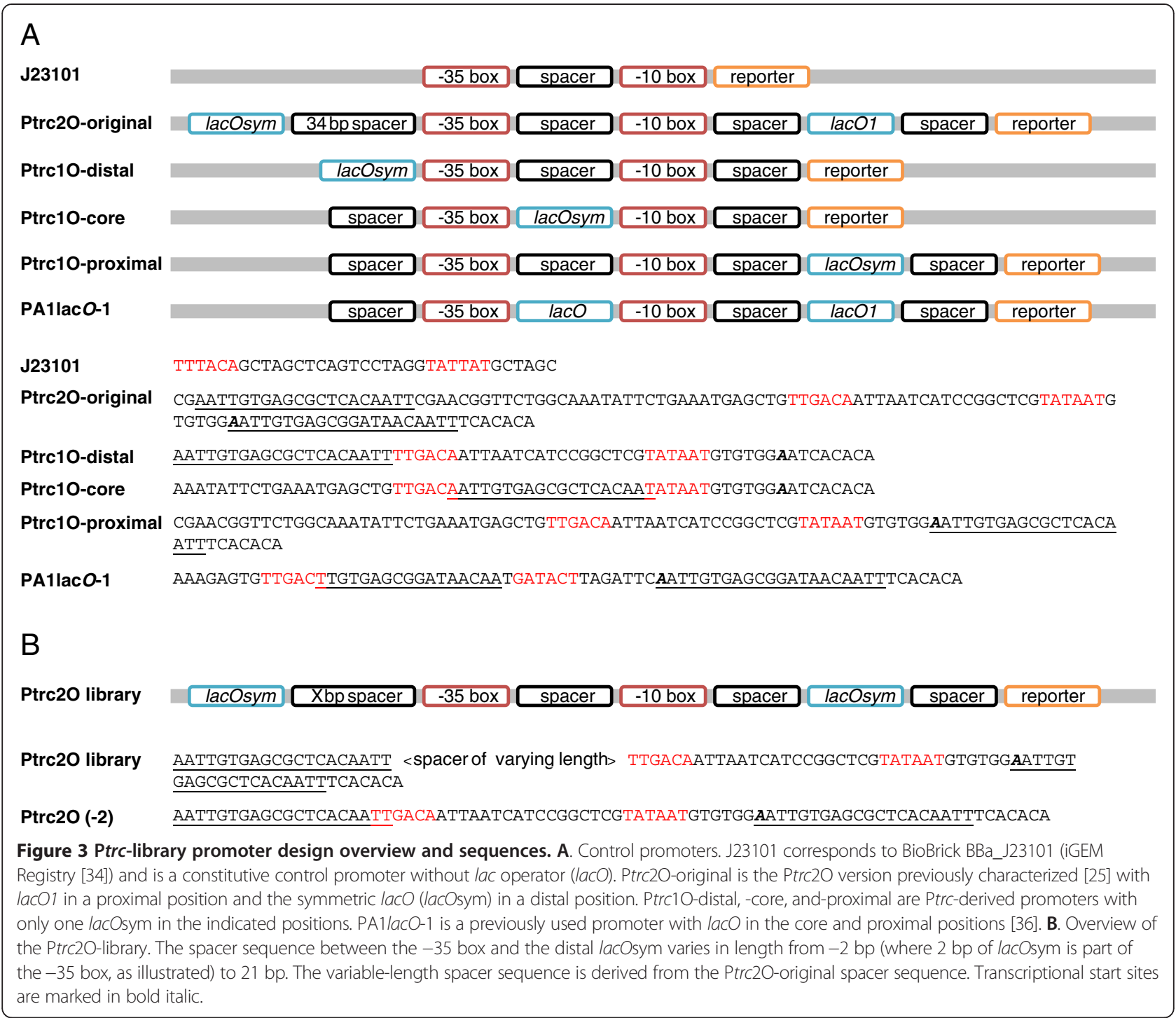

the about 6-fold stronger lacOsym operator [13]. Ptrc1Oproximal also serves as a proximal-lacOsym-only control for the Ptrc2O-library. The Ptrc2O-library was built based on Ptrc2O-original but, identically to Ptrc1O-proximal, the proximal lacO1 has been exchanged with lacOsym to enhance repression, and the distance between the primary proximal and the secondary distal lacOsym was varied by varying the length of a spacer sequence between the -35 element and the distal lacOsym. This spacer sequence varies in length from $-2 \mathrm{bp}$, where the distal lacOsym goes $2 \mathrm{bp}$ into the -35 element (Figure 3B), and $21 \mathrm{bp}$, and consists of the sequence previously used in the $34 \mathrm{bp}$ spacer of Ptrc2O-original. For characterization, the Ptrcderived promoter library was cloned with the same EYFP reporter construct as the constitutive promoters.

The Ptrc-derived promoter library was characterized in the E. coli NEB5 $\alpha \mathrm{F}^{\prime}$ lacl ${ }^{\mathrm{q}}$ strain (New England Biolabs) using a setup with LB medium in 96-well plates supplemented with relevant antibiotics and $0 \mathrm{mM}$ or $1 \mathrm{mM}$ IPTG, for non-induced and induced conditions, respectively. For the control promoters (Figure 4A), we could observe that the constitutive control BBa_J23101 displays stable expression under both induced conditions and non-induced. Ptrc2O-orig and Ptrc1O-prox display the same limited repression as previously characterized [25] where Ptrc2O-orig is more strongly repressed than Ptrc1O-prox. The distal lacOsym control promoter Ptrc1O-dist displays no detectable repression whereas Ptrc1O-core displays repression similar to Ptrc1O-prox. PA1lacO-1, which contains a core and a proximal lacO, displays strong repression yet highly inducible expression as expected from its original characterization [36]. For the Ptrc2O-library (Figure 4B), repression is a periodic function of the distance between the two lacOsym with a period of $11 \mathrm{bp}$, approximately the expected bp per turn of the chromatin DNA in vivo [20], for both the non- 


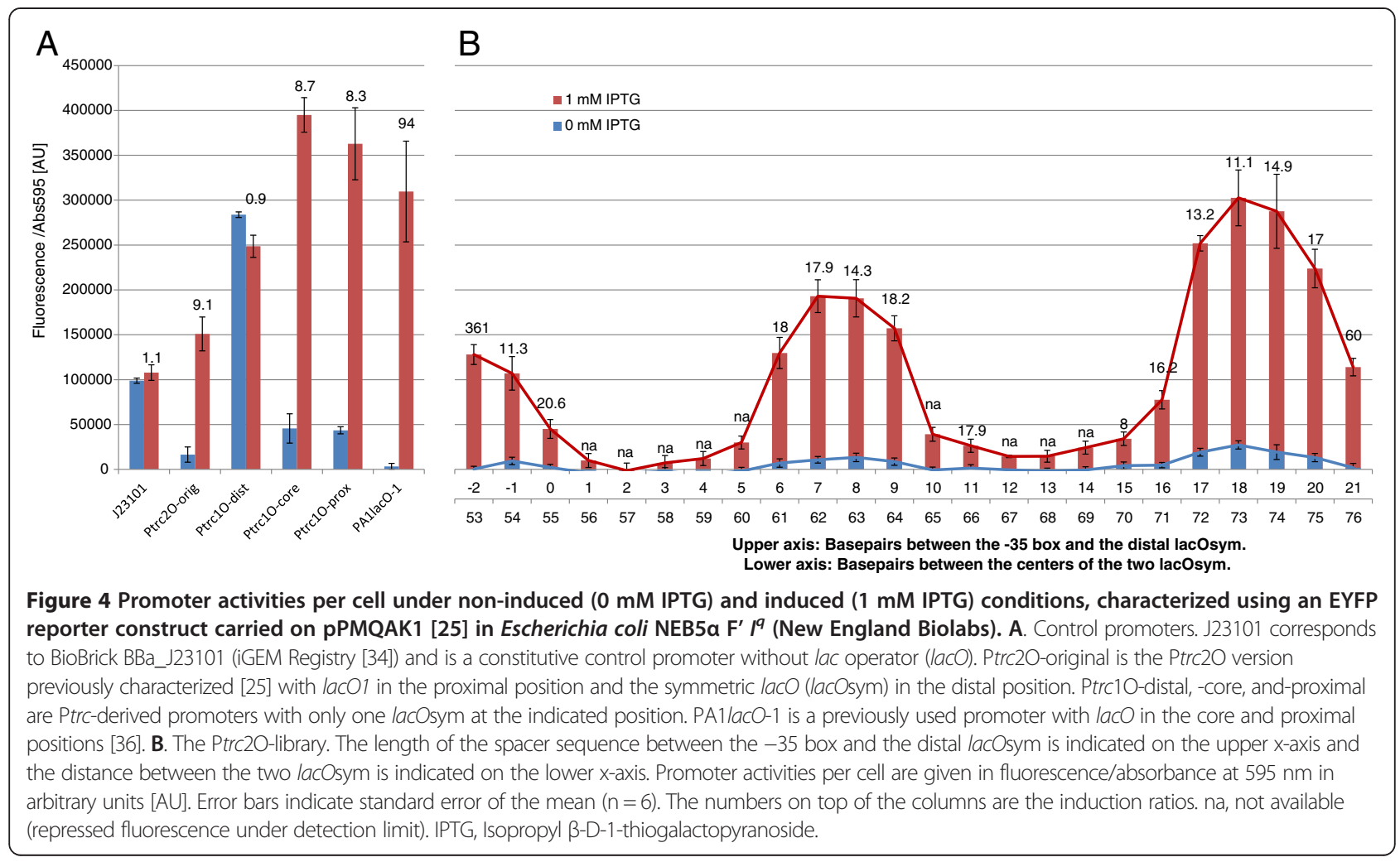

induced and the induced conditions. Maximal repression is represented by the two troughs of the pattern, separated by one turn of DNA or $11 \mathrm{bp}$, and minimal repression is represented by the two peaks of the pattern, also separated by one turn of DNA. Maximal repression is not observed for inter-lacOsym distances that are even multiples of $11 \mathrm{bp}$, corresponding to 55 and $66 \mathrm{bp}$, which is expected to be optimal if the average number per turn over the promoter sequence was $11 \mathrm{bp}$. Instead, repression maxima appear at 57 and 67 or $68 \mathrm{bp}$, a deviation of $2 \mathrm{bp}$ for the shorter loop and 1-2 bp for the longer one. Another observed trend for both conditions is that repression decreases with the distance of the secondary distal lacOsym from the promoter and the primary proximal lacOsym. The induction ratio values (Figure 4A and B) should be interpreted with caution for highly repressed promoters, as these values are close to the detection limit and even small variation due to noise will give large effects on the ratio.

\section{Characterization of the Ptrc-derived promoter library in the Lacl-expressing strain of Synechocystis}

The complete Ptrc-derived promoter library (Figure 3A and B) was characterized in the LacI-expressing strain of Synechocystis using a setup with BG11 medium in 96-well plates supplemented with relevant antibiotics and $0 \mathrm{mM}$ or $1 \mathrm{mM}$ IPTG, for non-induced and induced conditions, respectively. As a control for promoter activity in the absence of LacI, selected promoters (BBa_J23101, Ptrc 1O-core, PA1lacO-1 and the Ptrc2O-library constructs Ptrc2O-2, 13 and 18) were characterized also in the Chloramphenicol resistance-only strain of Synechocystis. This was done similarly as the characterization in the LacIexpressing strain of Synechocystis, but a setup with BG11 in $13 \mathrm{~mL}$ growth tubes was used instead of 96-well plates. For the control promoters (Figure 5A) we could observe that the constitutive control $\mathrm{BBa} \_$J23101 displays stable expression under both non-induced and induced conditions. Further, when comparing BBa_J23101-driven expression between the constitutive promoter assay (Figure 1) and the LacI-repression assay (Figure 5A), we observe that the level of fluorescence per cell is very similar even though the conditions were different. Wild-type Synechocystis was used in the first case whereas the LacIenhanced and the Chloramphenicol resistance-only strains were used in the second, the starter seed cultures were treated differently in the two cases, and $5 \mathrm{ml} 6$-well plate cultures were used in the first assay whereas $200 \mu$ l 96well plate cultures or $1 \mathrm{~mL}$ cultures in $13 \mathrm{~mL}$ growth tubes were used in the second. Hence, under the conditions tested here, BBa_J23101 is stably constitutive in Synechocystis. For Ptrc2O-orig we largely reproduced our previous characterization result [25], even though the assays differed in the expression of LacI and the growth conditions. There is a small difference in that Ptrc2O-orig is not as well-repressed here, which could indicate that the 


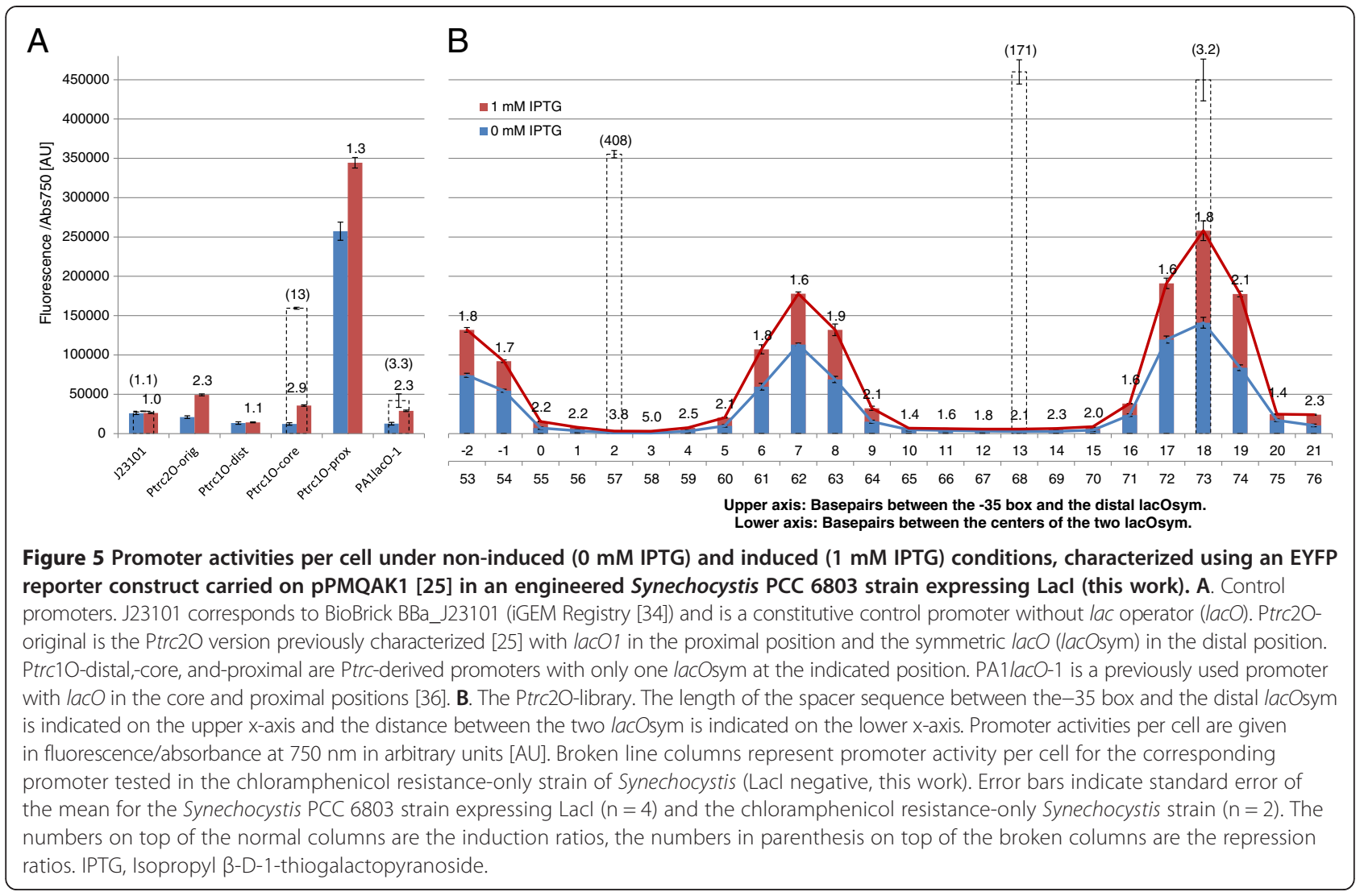

levels of LacI are somewhat lower than in our previous study. The Ptrc1O-prox, which differs from the Ptrc we used previously [25] in that lacO1 of Ptrc has been switched for the more efficient lacOsym, largely behaves like Ptrc under non-induced and induced conditions, being very poorly repressed. This contrasts with the situation in $E$. coli where it is more efficiently repressed. The distal lacOsym promoter Ptrc1O-dist does not show strong activity, whereas it does in E. coli, and displays no difference between non-induced or induced conditions, there agreeing with the E. coli results. The core lacOsym promoter Ptrc1O-core behaves very similarly to PA1lacO-1, which carries one slightly truncated $l a c O$ in the core and lacO1 in the proximal position, both displaying limited repression and induction. This contrasts strongly with the situation in E. coli where PA1lacO-1 is highly repressed yet displays strong expression under induction. When tested without LacI in the Chloramphenicol resistanceonly strain of Synechocystis, Ptrc1O-core displays 13-times higher activity than under LacI-repressed conditions. This shows that the lack of induction in Synechocystis is not due to an inherently weak Ptrc1O-core promoter but due to the presence of induced LacI. Still, the activity of Ptrc1O-core is weaker than that of Ptrc1O-prox, even when comparing Ptrc1O-core without LacI and Ptrc1Oprox with non-induced LacI (Figure 5B). However in
E. coli, Ptrc1O-core and Ptrc1O-prox are very similar under both repressed and induced conditions (Figure 4A). PA1lacO-1 however, displays a relatively low activity in Synechocystis even when LacI is absent (Figure 5A), and hence cannot be expected to be induced to higher levels. For the Ptrc2O-library (Figure 5B), the repression pattern is similar to the situation in E. coli, displaying periodic repression as a function of the distance between the two lacOsym with a period of $11 \mathrm{bp}$. Also as in E. coli, maximal repression can be observed for the troughs in the periodic repression pattern, minimal repression is represented as the two expression peaks separated by $11 \mathrm{bp}$ at the same positions as in E. coli, and repression efficiency decreases with the distance of the secondary distal lacOsym to the promoter and the primary proximal operator. However, even though repression of the Ptrc2O-library is very effective under both non-induced and induced conditions for optimal lacOsym positions, it is very ineffective in the least optimal positions represented by the expression peaks, even under non-induced conditions. This differs strongly from the more efficiently repressed nonoptimal distal lacOsym positions in E. coli, which, even though they are clearly visible as expression peaks, are repressed even under non-induced conditions. Further, repression in the geometrically-favored configurations is represented in Synechocystis by much wider troughs than 
in E. coli, for both conditions. This contrasts with the most unfavorable looping-geometries, represented by the narrower expression peaks in Synechocystis than in E. coli. To test if the very efficient repression for favorable DNA-looping geometries in Synechocystis is due to non LacI-related promoter inhibition of the Ptrc2O-library, we characterized the activity of Ptrc2O-2 and 13 in the absence of LacI in the Chloramphenicol resistance-only strain. These two highly repressed promoters, which are located in the centers of the repression troughs, display very high promoter activity in the absence of LacI (Figure 5B). Hence, they work as very high dynamic range LacI-regulated promoters, if expression could only be efficiently induced. Conversely, to test if the very non-efficient repression for non-favorable DNA-looping geometries in Synechocystis is due to non LacI-related promoter activation effects on the Ptrc2O-library, we also characterized the activity of Ptrc2O-18 in the absence of LacI. This inefficiently repressed promoter displayed a close to identical activity to the Ptrc2O-13 promoter, illustrating that the lack of repression of the Ptrc2O-library for non-favorable DNA-looping geometries in Synechocystis is not due to inherently stronger promoters. Taken together, these results show that the periodical repression pattern of the Ptrc2O-library in the LacI-expressing strain of Synechocystis is due to LacI-facilitated DNA-looping.

\section{Characterization of the proximal lacO-library in E. coli DH5aZ1 and Synechocystis}

Differences in how the E. coli and the Synechocystis RNAP interact with LacI bound to the proximal region of trcderived promoters could be investigated by comparing IPTG-induction or LacI-repression patterns of a proximal lacO-library between E. coli and Synechocystis. Because induction or repression ratios of the same promoters are studied, expression differences between promoters caused by differences in the $5^{\prime}$-UTRs will be cancelled, and the ratios will reflect the efficiencies of LacI repression for specific promoters. Such induction or repression ratios of promoter libraries where the position of single lac operators relative to the promoter was varied by single basepairs have been studied before to investigate variation in LacI repression $[19,20]$. We reason that a potential difference in the DNA-binding activity between the E. coli and the Synechocystis RNAP might be visible in differences in the pattern of this ratio. Therefore, we designed a promoter library in which we vary the distance between the center of a symmetric lacO and the TSS of a Ptrc core promoter from -2 to +18 bp by adjusting the length of a spacer in 1 bp steps and from +18 to +24 bp in 2 bp steps (Table 1). We inserted this proximal lacO-library into an E. coli strain that expresses high amounts of LacI repressor, DH5 $\alpha \mathrm{Z1}$, to enable the calculation of IPTG-induction ratios, and into both the LacI-expressing Synechocystis strain and the chloramphenicol resistance-only strain, to enable the calculation of both IPTG-induction and LacIrepression ratios.

The characterization of the proximal lacO-library in E. coli $\mathrm{DH} 5 \alpha \mathrm{Z} 1$ was performed similarly to the characterization of the Ptrc-derived promoter library in $\mathrm{NEB} 5 \alpha$, but a key difference is that the proximal lacOlibrary was followed in time by measuring the same plates at different time-points and then continuing the experiment. The induction ratios were calculated from the fluorescence/absorbance values of the induced/noninduced cultures and the standard errors of the ratios were estimated (Figure 6A). An apparent steady-state of fluorescence/cell for most of the promoters (data not shown) was observed at ca 3.8 hours after the start of the experiment. At this time point, the induction ratios are also the highest; the J23101 constitutive control has reached its expected ratio of ca 1 and the ratios diminish progressively in value at later time-points. The induction ratio pattern established from ca 3.8 hours remains, however, also at later time points (Figure 6A). As shown in previous studies of induction and repression ratios of libraries of proximal lacO-promoters in $E$. coli [19], the lacO centered at +11 relative to the TSS (prox-11 in Figure 6A) provides the most efficient repression, closely followed by the +10 promoter. The +11 position corresponds to the position of lacO1 in the wild-type E. coli lac promoter [10] and is one helix turn away from the TSS, putting LacI on the same side of the DNA as the RNAP.

The characterization of the proximal lacO-library in Synechocystis was performed similarly to the characterization of the Ptrc-derived promoter library, but $13 \mathrm{~mL}$ growth tubes were used instead of 96-well plates, and, as compared with the E. coli DH5 $\alpha \mathrm{Z} 1$ experiment, only one time-point was used. The induction ratios were calculated from the fluorescence/absorbance values of the induced/non-induced LacI-expressing Synechocystis cultures and the standard errors of the ratios were estimated (Figure 6B). The repression ratios were calculated from the fluorescence/absorbance values of the chloramphenicol resistance-only cultures/non-induced LacI-expressing Synechocystis cultures and the standard errors of the ratios were estimated (Figure 6C). First, we observe that the ratios are much smaller, with a smaller difference between the different promoters, than in $E$. coli DH5 $\alpha \mathrm{Z1}$. However, both the induction and the repression ratios in Synechocystis are similar to the induction ratios in E. coli DH5 $\alpha \mathrm{Z} 1$. Also in Synechocystis, the centered +11 position of the $l a c O$ relative to the TSS produces the most efficiently repressed proximal lacO promoter. To summarize, no difference in the induction or repression patterns between E. coli and Synechocystis can be detected. 
Table 1 Promoter DNA sequences

\begin{tabular}{l} 
Promoter nam \\
\hline BBa_J23100 \\
BBa_J23101 \\
BBa_J23102 \\
BBa_J23105 \\
BBa_J23106 \\
BBa_J23109 \\
BBa_J23113 \\
BBa_J23114 \\
PnirA
\end{tabular}

PpetE

PrnpB

Ptrc2O-(-2)

Ptrc2O-(-1)

Ptrc2O-0

Ptrc2O-1

Ptrc2O-2

Ptrc2O-3

Ptrc2O-4

Ptrc2O-5

Ptrc2O-6

Ptrc2O-7

Ptrc2O-8

Ptrc2O-9

Ptrc2O-10

\section{Sequence $5^{\prime}$ to $3^{\prime}$}

TTGACGGCTAGCTCAGTCCTAGGTACAGTGCTAGC

TITACAGCTAGCTCAGTCCTAGGTATTATGCTAGC

TTGACAGCTAGCTCAGTCCTAGGTACTGTGCTAGC

TITACGGCTAGCTCAGTCCTAGGTACTATGCTAGC

TITACGGCTAGCTCAGTCCTAGGTATAGTGCTAGC

TITACAGCTAGCTCAGTCCTAGGGACTGTGCTAGC

CTGATGGCTAGCTCAGTCCTAGGGATTATGCTAGC

TITATGGCTAGCTCAGTCCTAGGTACAATGCTAGC

CAAGCTCAGAATGCTGCGGGGAGAAGGGCAACTCCC

CACCAGCCCCAAATTTITGCTGGCGATAAATATTTTTCGGTTTAATTGTTCACA

AAGCTTTTTGAATTTGAGTTATAGAAATTTATTGGCTGG

TAATGCTITITTGCCCCCCTGCTGGACTTCATTGATCCTTGCCTATAC

CATCAATATCATTGGTCAATAATGATGATGATTGACTAAAACATGTT

TAACAAAATTTAACGCATATGCTAAATGCGTAAACTGCATATGCCTT GGCTGAGTGTAATTTACGTTACAAATTTTAACGAAACGGGAACCCT ATATTGATCTCTACTGTTATCTGGCT

TCATAGCGGTTGCCCAATCTAACTCAGGGAGCGACTTCAGCCCAC AAAAAACACCACTGGGCCTACTGGGCTATTCCCATTATCATCTACA TTGAAGGGATAGCAAGCTAATITTATGACGGCGATCGCCAAAAAC AAAGAAAATTCAGCAATTACCGTGGGTAGCAAAAAATCCCCATCTAA AGTTCAGTAAATATAGCTAGAACAACCAAGCATTITCGGCAAAGTAC TATTCAGATAGAACGAGAAATGAGCTTGTTCTATCCGCCCGGGGCTG AGGCTGTATAATCTACGACGGGCTGTCAAACATTGTGATACCATGGG CAGAAGAAAGGAAAAACGTCCCTGATCGCCTIITTGGGCACGGAGT AGGGCGTTACCCCGGCCCGTTCAACCACAAGTCCCTATAGATACAATCGCCAAGAAGT

TTCAATGCGGTCCAATACCTCCCCTGCCCAACTGGGTAAGCTCGCGGC TCCACTGAGTAATACAGACAAGGCTAAACAGGCAAATTITTTCATTGG TCAACTCCTAGCACCAATTTCCCAAGACTACGGAGGGGGCAATGAAGTT TCAATTAATTGGGGTCACAAACCACAGCGGCCTATGGCTCTAATCAATGGCACACTAGAAAAA

AATTGTGAGCGCTCACAATTGACAATTAATCATCCGGCTCGTATAATGTGTGGAATTGTGAG CGCTCACAATTTCACACA

AATTGTGAGCGCTCACAATTTGACAATTAATCATCCGGCTCGTATAATGTGTGGAATTGTGA GCGCTCACAATTTCACACA

AATTGTGAGCGCTCACAATTTGACAATTAATCATCCGGCTCGTATAATGTGTGGAATTGT GAGCGCTCACAATTTCACACA

AATTGTGAGCGCTCACAATTGTTGACAATTAATCATCCGGCTCGTATAATGTGTGGAATT GTGAGCGCTCACAATTTCACACA

AATTGTGAGCGCTCACAATTTGTTGACAATTAATCATCCGGCTCGTATAATGTGTGGAATT GTGAGCGCTCACAATTTCACACA

AATTGTGAGCGCTCACAATTCTGTTGACAATTAATCATCCGGCTCGTATAATGTGTGGAA TTGTGAGCGCTCACAATTTCACACA

AATTGTGAGCGCTCACAATTGCTGTTGACAATTAATCATCCGGCTCGTATAATGTGTGG AATTGTGAGCGCTCACAATTTCACACA

AATTGTGAGCGCTCACAATTAGCTGTTGACAATTAATCATCCGGCTCGTATAATGTGTGG AATTGTGAGCGCTCACAATTTCACACA

AATTGTGAGCGCTCACAATTGAGCTGTTGACAATTAATCATCCGGCTCGTATAATGTGTG GAATTGTGAGCGCTCACAATTTCACACA

AATTGTGAGCGCTCACAATTTGAGCTGTTGACAATTAATCATCCGGCTCGTATAATGTGT GGAATTGTGAGCGCTCACAATTTCACACA

AATTGTGAGCGCTCACAATTATGAGCTGTTGACAATTAATCATCCGGCTCGTATAATGTG TGGAATTGTGAGCGCTCACAATTTCACACA

AATTGTGAGCGCTCACAATTAATGAGCTGTTGACAATTAATCATCCGGCTCGTATAATGTG TGGAATTGTGAGCGCTCACAATTTCACACA

AATTGTGAGCGCTCACAATTAAATGAGCTGTTGACAATTAATCATCCGGCTCGTATAATGT GTGGAATTGTGAGCGCTCACAATTTCACACA 
Table 1 Promoter DNA sequences (Continued)

\begin{tabular}{|c|c|}
\hline Ptrc2O-11 & $\begin{array}{l}\text { AATTGTGAGCGCTCACAATTGAAATGAGCTGTTGACAATTAATCATCCGGCTCGTATAATGTGTGGAATTGT } \\
\text { GAGCGCTCACAATTTCACACA }\end{array}$ \\
\hline Ptrc2O-12 & $\begin{array}{l}\text { AATTGTGAGCGCTCACAATTTGAAATGAGCTGTTGACAATTAATCATCCGGCTCGTATAATGTGTGGAATTG } \\
\text { TGAGCGCTCACAATTTCACACA }\end{array}$ \\
\hline Ptrc2O-13 & $\begin{array}{l}\text { AATTGTGAGCGCTCACAATTCTGAAATGAGCTGTTGACAATTAATCATCCGGCTCGTATAATGTGTGGAATT } \\
\text { GTGAGCGCTCACAATTTCACACA }\end{array}$ \\
\hline Ptrc2O-14 & $\begin{array}{l}\text { AATTGTGAGCGCTCACAATTTCTGAAATGAGCTGTTGACAATTAATCATCCGGCTCGTATAATGTGTGGAAT } \\
\text { TGTGAGCGCTCACAATTTCACACA }\end{array}$ \\
\hline Ptrc2O-15 & $\begin{array}{l}\text { AATTGTGAGCGCTCACAATTTCTGAAATGAGCTGTTGACAATTAATCATCCGGCTCGTATAATGTGTGGAA } \\
\text { TTGTGAGCGCTCACAATTCACACA }\end{array}$ \\
\hline Ptrc2O-16 & $\begin{array}{l}\text { AATTGTGAGCGCTCACAATTATTCTGAAATGAGCTGTTGACAATTAATCATCCGGCTCGTATAATGTGTGGA } \\
\text { ATTGTGAGCGCTCACAATTTCACACA }\end{array}$ \\
\hline Ptrc2O-17 & $\begin{array}{l}\text { AATTGTGAGCGCTCACAATTAATTCTGAAATGAGCTGTTGACAATTAATCATCCGGCTCGTATAATGTGTGG } \\
\text { AATTGTGAGCGCTCACAATTTCACACA }\end{array}$ \\
\hline Ptrc2O-18 & $\begin{array}{l}\text { AATTGTGAGCGCTCACAATTATATTCTGAAATGAGCTGTTGACAATTAATCATCCGGCTCGTATAATGTGTG } \\
\text { GAATTGTGAGCGCTCACAATTCACACA }\end{array}$ \\
\hline Ptrc2O-19 & $\begin{array}{l}\text { AATTGTGAGCGCTCACAATTAATATTCTGAAATGAGCTGTTGACAATTAATCATCCGGCTCGTATAATGTGT } \\
\text { GGAATTGTGAGCGCTCACAATTTCACACA }\end{array}$ \\
\hline Ptrc2O-20 & $\begin{array}{l}\text { AATTGTGAGCGCTCACAATTAAATATTCTGAAATGAGCTGTTGACAATTAATCATCCGGCTCGTATAATGTG } \\
\text { TGGAATTGTGAGCGCTCACAATTTCACACA }\end{array}$ \\
\hline Ptrc2O-21 & $\begin{array}{l}\text { AATTGTGAGCGCTCACAATTCAAATATTCTGAAATGAGCTGTTGACAATTAATCATCCGGCTCGTATAATGT } \\
\text { GTGGAATTGTGAGCGCTCACAATTTCACACA }\end{array}$ \\
\hline Ptrc2O-orig & $\begin{array}{l}\text { CGAATTGTGAGCGCTCACAATTCGAACGGTTCTGGCAAATATTCTGAAATGAGCTGTTGACAATTAATCATC } \\
\text { CGGCTCGTATAATGTGTGGAATTGTGAGCGGATAACAATTTCACACA }\end{array}$ \\
\hline Ptrc10-dist & AATTGTGAGCGCTCACAATTTGACAATTAATCATCCGGCTCGTATAATGTGTGGAATCACACA \\
\hline Ptrc1O-core & AAATATTCTGAAATGAGCTGTTGACAATTGTGAGCGCTCACAATATAATGTGTGGAATCACACA \\
\hline Ptrc10-prox & $\begin{array}{l}\text { CGAACGGTTCTGGCAAATATTCTGAAATGAGCTGTTGACAATTAATCATCCGGCTCGTATAATGTGTGGAA } \\
\text { TTGTGAGCGCTCACAATTTCACACA }\end{array}$ \\
\hline $\mathrm{PA} 1 / \mathrm{acO}-1$ & AAAGAGTGTTGACTTGTGAGCGGATAACAATGATACTTAGATTCAATTGTGAGCGGATAACAATTCACACA \\
\hline PtrcOs-prox-8 & TTGACAATTAATCATCCGGCTCGTATAATGTGTAATTGTGAGCGCTCACAATT \\
\hline PtrcOs-prox-9 & TTGACAATTAATCATCCGGCTCGTATAATGTGTGAATTGTGAGCGCTCACAATT \\
\hline PtrcOs-prox-10 & TTGACAATTAATCATCCGGCTCGTATAATGTGTGGAATTGTGAGCGCTCACAATT \\
\hline PtrcOs-prox-11 & TTGACAATTAATCATCCGGCTCGTATAATGTGTGGAAATTGTGAGCGCTCACAATT \\
\hline PtrcOs-prox-12 & TTGACAATTAATCATCCGGCTCGTATAATGTGTGGAGAATTGTGAGCGCTCACAATT \\
\hline PtrcOs-prox-13 & TTGACAATTAATCATCCGGCTCGTATAATGTGTGGAGTAATTGTGAGCGCTCACAATT \\
\hline PtrcOs-prox-14 & TTGACAATTAATCATCCGGCTCGTATAATGTGTGGAGTCAATTGTGAGCGCTCACAATT \\
\hline PtrcOs-prox-15 & TTGACAATTAATCATCCGGCTCGTATAATGTGTGGAGTCGAATTGTGAGCGCTCACAATT \\
\hline PtrcOs-prox-16 & TTGACAATTAATCATCCGGCTCGTATAATGTGTGGAGTCGTAATTGTGAGCGCTCACAATT \\
\hline PtrcOs-prox-17 & TTGACAATTAATCATCCGGCTCGTATAATGTGTGGAGTCGTCAATTGTGAGCGCTCACAATT \\
\hline PtrcOs-prox-18 & TTGACAATTAATCATCCGGCTCGTATAATGTGTGGAGTCGTCCAATTGTGAGCGCTCACAATT \\
\hline PtrcOs-prox-20 & TTGACAATTAATCATCCGGCTCGTATAATGTGTGGAGTCGTCCAGAATTGTGAGCGCTCACAATT \\
\hline PtrcOs-prox-22 & TTGACAATTAATCATCCGGCTCGTATAATGTGTGGAGTCGTCCAGACAATTGTGAGCGCTCACAATT \\
\hline PtrcOs-prox-24 & TTGACAATTAATCATCCGGCTCGTATAATGTGTGGAGTCGTCCAGACTCAATTGTGAGCGCTCACAATT \\
\hline
\end{tabular}

\section{Discussion}

To elucidate the design principles of LacI-repressed promoters and DNA-looping in cyanobacteria, we constructed a Ptrc-derived promoter library consisting of single lac operator control promoters (Figure 3A) and dual lacO promoters with LacI-facilitated DNA-loop capability
(Figure 3B). For characterizing the repression and induction of this library, we first characterized several artificial minimal promoters in Synechocystis and used this knowledge to engineer a constitutive LacI-expressing strain of Synechocystis. Finally, to investigate if differences between the E. coli and Synechocystis RNA polymerases would 


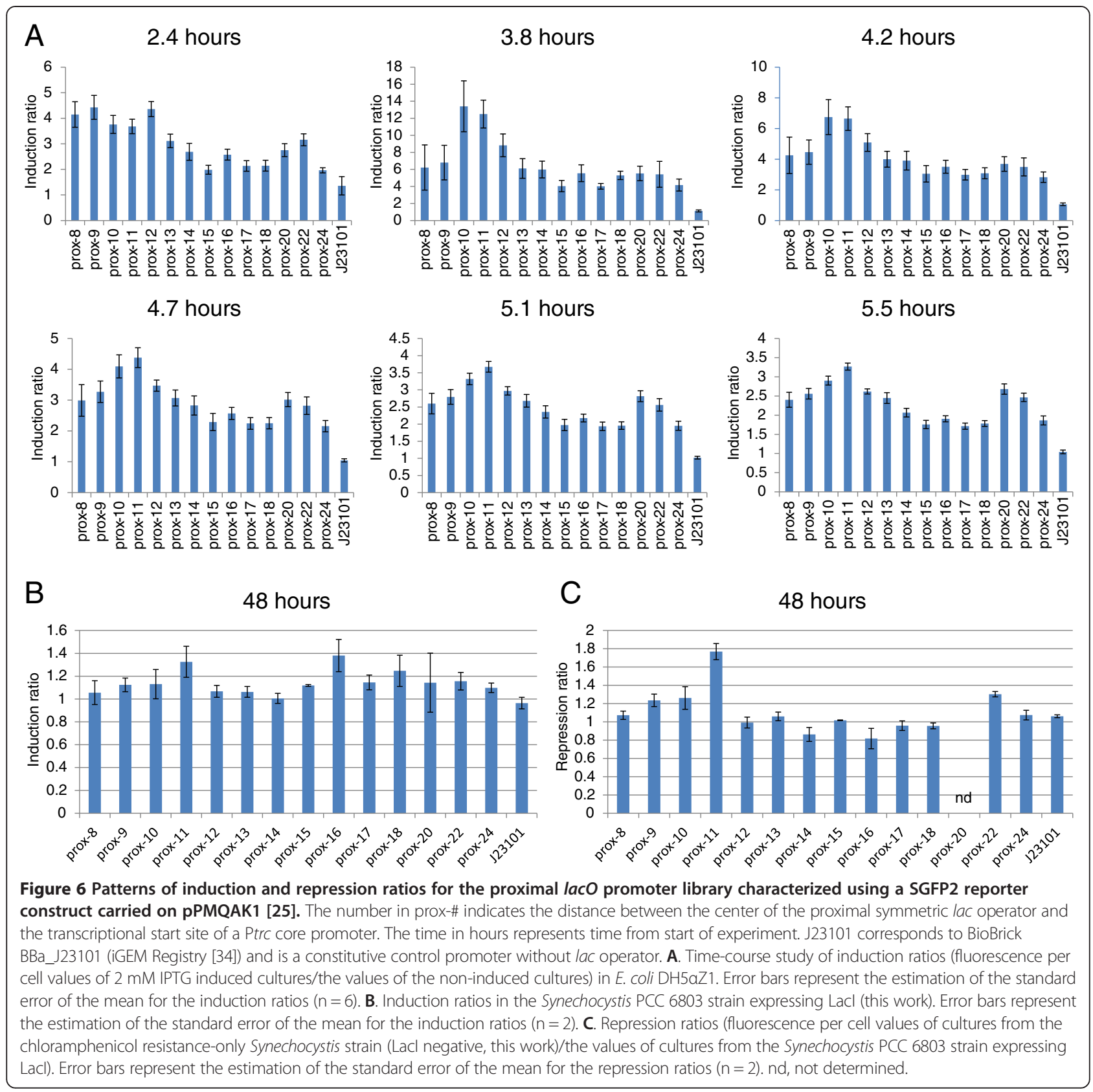

affect LacI-repression of promoters with proximally located $l a c O$, we engineered and characterized a library of proximal lacO promoters in both E. coli and Synechocystis.

\section{Minimal and artificial promoters as orthogonal alternatives to native cyanobacterial promoters} As orthogonal parts can help minimize or eliminate crosstalk and unwanted interactions between parts or between parts and the chassis [7], we first set out to characterize several minimal and artificial promoters that could potentially drive the constitutive expression of LacI in
Synechocystis. The artificial, minimal and constitutive promoters $\quad \mathrm{BBa} \_$23100, $\quad \mathrm{BBa} \_$23101, $\quad \mathrm{BBa} \_J 23102$, BBa_J23105, BBa_J23106, BBa_J23109, BBa_J23113 and $\mathrm{BBa} \_23114$ were found to span a greater range of expression levels, high and low, as compared to the three commonly used native promoters PnirA, PpetE and PrnpB from Synechocystis. The artificial promoters are derived from a consensus $E$. coli promoter, which -35 and -10 elements are almost identical to the consensus -35 and -10 boxes of Synechocystis [37]. These types of promoters, belonging to the group 1 promoters in cyanobacteria, are 
served by the primary cyanobacterial sigma factor SigA during normal growth conditions [26]. Hence, all the artificial promoters tested here can be classified as cyanobacterial group 1 promoters, and as orthogonal and minimal promoters, minimizing the risk for cross-talk with native transcription factors or sRNAs, they are expected to remain constitutive under normal growth conditions. In favor of this, the BBa_23101 promoter was found to display stable levels of expression of EYFP per cell under the different conditions of light-intensity, culture volumes and mixing, presence of the exogenous chemical IPTG, and three chassis (wild-type, LacI-enhanced and chloramphenicol resistance-only strains of Synechocystis) that were used in this study. Further, the weak BBa_J23114 promoter was used with RBS* to express relatively low levels of LacI/cell (about 190 tetramers of LacI/cell) in the LacI-enhanced strain of Synechocystis. Therefore, we suggest that promoters from the iGEM Registry's Anderson collection could be used as minimal orthogonal alternatives to native promoters for constitutive expression in cyanobacteria, or as core promoters for the engineering of regulated promoters. Further, as the BBa_J23101 promoter has been used as a standard reference promoter in E. coli [38], we propose that it could fulfill the same role in cyanobacteria. As all the Ptrc-derived promoters used in this study are close-toconsensus group 1 cyanobacterial promoters, orthogonal to Synechocystis, and minimal in the sense that they only contain the necessary elements for recognition by RNAP and regulation by LacI, they are also expected to be constitutive in the absence of LacI, which we showed herein for selected members of the library. The orthogonality also means that they should display no or minimal cross-talk with native transcription factors and sRNAs. However, to truly test the variation of expression, and find constitutive promoters with minimal variation of expression, a more rigorous study of diverse environmental conditions than the present work is warranted. For example, cyanobacteria naturally grow in day and night cycles and have a circadian rhythm that affects a large part of the transcriptome, seemingly mainly through modulating DNA topology [39]. How or if circadian rhythms would modulate orthogonal promoters in cyanobacteria would have to be tested. Further, a change in DNA topology could change the relative spatial positioning or phasing on the helix of a promoter and the binding site of a transcription factor, such as LacI, possibly rendering repression even by an orthogonal repressor circadian rhythm-dependent.

Other environmental conditions are also relevant to test for orthogonal promoters, such as higher variations in light intensity and duration of darkness, changes in salinity and temperature. It is well known that different environmental variations give rise to sigma-factor switching, a phenomenon that helps cyanobacteria adapt to diverse changes by switching between different sigma-factors that activate the expression of stress responses [26]. To further decouple synthetic circuits from cell regulation and phenomena such as sigma-switching, system orthogonality could be increased by using orthogonal RNAP and their corresponding promoters, which are decoupled from the native transcriptional machinery of the host. For example, the bacteriophage T7 RNAP can be engineered to produce orthogonal genetic circuits incorporating several mutually orthogonal T7 RNAP [40] or orthogonal split T7 RNAP [41].

\section{Lacl-repressed promoters in E. coli and Synechocystis}

Since the following analysis builds on comparisons of promoter repression patterns, it is important to note that expression also depends on the $5^{\prime}$ untranslated region, the coding sequence of the particular gene of interest (GOI) and their combined interactions. The folding status of the 5' UTR and in particular the ribosome binding site (RBS) strongly affects translation initiation [42]. Since the first part of the GOI sequence is located close to the 5' UTR on the mRNA, its identity will affect potential mRNA secondary structures involving the RBS. Further, internal GOI sequence elements such as codon identities and secondary structures will affect translation elongation [43]. Consequently, when comparing promoter strengths indirectly through the expression of a reporter gene it only makes sense to compare constructs with the same 5' UTR and coding sequence. In this study, the same reporter cassette featuring the same RBS and coding sequence is used for all promoters within a library. However, some of the promoters in the Ptrc-derived library differ in the sequences that come after the transcription start site (TSS), meaning that these promoters will have slightly different 5' UTRs (Figures 3A and B). Among these promoters, Ptrc1O-proximal and the whole Ptrc2O-library, except Ptrc2O-original, share the same 5' UTR. Further, Ptrc1Odistal and Ptrc1O-core share the same 5' UTR whereas BBa_J23101, Ptrc2O-original and PA1lacO-1 do not share 5' UTRs. However, the induction ratios can still be compared between promoters that do not share exact 5' UTRs as $5^{\prime}$ UTR effects that affect both the induced state and the non-induced state will be cancelled. This is true also for the proximal lacO promoter library (Table 1), where all promoters differ slightly in their $5^{\prime}$ UTRs but only induction or repression ratios are compared. Finally, it should be noted that the fluorescence from promoters that are highly repressed are close to the detection limit, meaning that even small amounts of variation due to noise will affect the induction or repression ratio values strongly. Hence, in general caution should be exercised when comparing promoter activity ratios for strongly repressed promoters.

We engineered a strain of Synechocystis to express similar levels of LacI as in our previous study [25] for 
repression of the Ptrc-derived promoter library. Also, we previously observed that $\mathrm{Ptrc}$ and $\mathrm{Ptrc} 2 \mathrm{O}$ behave as constitutive promoters of approximately the same strength in the absence of heterologously expressed LacI, displaying stable expression levels irrespective of the presence of IPTG. Therefore, and since we are herein interested in analyzing the patterns of repression and induction depending on lacO location(s), we did not analyze the majority of the Ptrc-derived promoter library in the absence of LacI. Further, by comparing repression and induction levels of Ptrc2O (here termed Ptrc2O-original to distinguish it from the other Ptrc2O promoters) in Synechocystis between the present (Figure 5A) and our previous study [25], we observe a similar pattern but that the induction/ repression ratio is about two-fold lower in this study. As the two studies differ in both the characterization growth conditions and the LacI expression cassettes we cannot confidently conclude the reason for this difference, but the discrepancy leads us to estimate that the levels of LacI in the present study are similar to or lower than in our previous study. We estimated the number of LacI tetramers per cell to be about 190 for the engineered LacIexpressing strain developed here, so the number can be expected to be higher than 190 in our previous study [25].

To investigate if a lack of LacI binding the proximal lacO1 of Ptrc was the reason for the limited repression in Synechocystis that we previously observed [25], we exchanged lacO1 with the stronger lacOsym operator. However, no increase in the induction ratio was observed (Figure 5A). This however, could be due to the possibly lower levels of LacI in this study, or that the 1 bp shorter lacOsym places LacI in a spatially less optimal position relative to the promoter's TSS than lacO1. Nonetheless, when comparing the repression and induction results of Ptrc1O-proximal between E. coli (Figure 4A) and Synechocystis (Figure 5A) we managed to confirm our previous results [25] that the proximal lacO position downstream to and overlapping $1 \mathrm{bp}$ with the TSS leads to more efficient repression in E. coli as compared to in Synechocystis. Another study observed the same lack of repression of Ptrc in Synechocystis when using another construct to express LacI [28]. This reproducibility, and the fact that the levels of LacI expressed in the LacI-enhanced strain of Synechocystis are sufficient for much more effective repression of other $l a c O$ promoters (Figures 5A and B) lead us to hypothesize that the lack of repression of Ptrc and its twin Ptrc1Oproximal in Synechocystis is due to the characteristics of RNAP, and not due to the lack of LacI. In bacteria, the RNAP holoenzyme consists of the $\alpha_{2}, \beta, \beta^{\prime}$ and $\omega$ subunits plus a $\sigma$-factor that gives promoter specificity [44]. A time-resolved E. coli RNAP-promoter footprinting study showed that RNAP makes first contact with the upstream part of the promoter, and then extends downstream towards the -10 element, the TSS and all the way to about +20 bp from the TSS as it proceeds from a closed to an open complex [45]. It was recently shown that the RNAP clamp, consisting of pincer-like parts of the $\beta^{\prime}$ and the $\beta$ subunits, progressively closes around the double stranded DNA downstream from the TSS as the complex approaches transcription initiation, and then remains closed during elongation [46]. In cyanobacteria, the $\beta$ ' subunit has been split up in two parts: $\gamma$, which corresponds to the $\mathrm{N}$-terminal part of the bacterial $\beta$ ' subunit, and $\beta$,' which corresponds to the C-terminal part [31,32]. It was observed in a later study that enteric and cyanobacterial RNAP transcribe promoters differently, and differences in RNAP architecture, including a large insertion in the $\beta$ ' subunit was suggested to be the cause [47]. Since then, these differences between the enteric and the cyanobacterial RNAP has been suggested to explain differences in promoter activity due to single nucleotide mutations [48] and more recently differences in elongation rate and fidelity [49].

Hence we hypothesize that the differences between the E. coli RNAP $\beta$ ' subunit and the cyanobacterial RNAP $\gamma+\beta$ ' subunits could explain the apparent decreased sensitivity of Synechocystis RNAP towards LacI binding the proximal lac operator of Ptrc and Ptrc1O-proximal. Since the $\beta$ ' and $\beta$ clamp of enteric RNAP closes around the DNA downstream of the TSS during transcription initiation, and $\beta^{\prime}$ is the subunit that differs the most between cyanobacteria and $E$. coli, we speculate that the RNAP clamp of cyanobacteria may possess an increased stability or a different DNA footprint than enteric RNAP, blocking or removing a proximally bound LacI more effectively. If the DNA footprint differs, the proximal promoter binding site location for maximizing repression by a transcription factor is likely to be different for cyanobacteria as compared to enterobacteria. To test if the DNA footprint of E. coli and Synechocystis RNAP differ for the proximal promoter region close to and downstream the TSS, we designed a library of proximal lacO promoters in which we vary the distance between the center of a symmetric lacO and the TSS of a Ptrc core promoter by steps of 1 or $2 \mathrm{bp}$. We characterized the induction and/or the repression ratio patterns for this proximal promoter library in both E. coli and Synechocystis, but could not detect any differences. Hence, our results do not support the hypothesis that there is a difference in the DNA footprint between the E. coli and Synechocystis RNAP at the promoter proximal location. If the cyanobacterial RNAP possesses an increased DNA-binding stability in the promoter proximal region remains to be tested, and what the potential effect of the $\beta$ ' subunit split and insertion in cyanobacteria is remains to be shown. 
Finally, the fact that Ptrc can be more efficiently repressed and induced in Synechococcus elongatus PCC 7942 [27] than in Synechocystis [25,28] may simply be explained by a higher number of LacI/cell in the Synechococcus study than in the Synechocystis studies. Indeed, the numbers of LacI/cell expressed in the LacI-enhanced strain of Synechocystis herein presented is about five times lower than the per cell value for $E$. coli NEB5 $\alpha$, and about sixteen times lower than in DH5 $\alpha \mathrm{Z} 1$.

Recently, the in $E$. coli well-repressed yet highly inducible promoter PA1lacO-1 [36] was characterized in Synechocystis using a plasmid-carried lacl $^{q}$ gene for expressing LacI and an ethylene-forming enzyme (EFE) as a reporter [28]. Characterization was carried out by measuring the rate of ethylene formation per volume, time and cell density and comparing IPTG-inducing and non-inducing conditions for different cell densities. The repression of PA1lacO-1 was found to be higher at lower cell densities and lower at higher cell densities. Further, they tested Ptrc with the same setup and found that PA1lacO-1 could be induced to the same level as Ptrc. To enable the comparison of a regulated high dynamic range E. coli promoter with its activity in Synechocystis, we characterized PAllacO-1 with the same EYFP reporter construct as the rest of the Ptrc-derived library (Figures 3A and B). As expected, we found that PA1lacO-1 was strongly repressed yet highly inducible in E. coli (Figure 4A). However, even though we found that PA1lacO-1 is repressed in Synechocystis, in line with the previous study [28], we found that it could not be induced to the activity of Ptrc1O-prox (Figure 5A), which is almost identical in its repression and induction behavior to Ptrc [25]. Still, it is problematic to directly compare Ptrc1O-prox/Ptrc with PA1lacO-1 since they have slightly different $5^{\prime}$ UTRs, as well as comparing the two studies that use different reporters, both factors potentially affecting expression. Further, for promoter activity quantification in general, it is problematic to infer promoter activities indirectly from the expression of protein reporters. This problem can be solved by directly measuring promoter mRNA output using quantitative reverse transcription PCR. However, this poses a problem of its own as it cannot be done in living cells. Hence, it may sometimes still be preferable to characterize promoters indirectly through the use of fluorescent reporters, since this can be done in vivo. For instance, absolute quantification of promoters can be done using fluorescent protein reporters together with quantitative models to derive promoter strengths [50] or the number of RNAP that clears the promoter per second [38]. Finally, using the activity of an enzymatic protein reporter for inferring promoter activity adds an additional level of uncertainty over using fluorescent proteins, as enzymes depend on many factors, for instance substrate availability.
These considerations make it difficult to speculate about the reason(s) for the difference in induced expression of Ptrc1O-prox/Ptrc versus PA1lacO-1 when comparing this and the previous study [28]. Further, PA1lacO-1 consists of a proximal lacO1 $1 \mathrm{bp}$ downstream in position as compared to Ptrc and Ptrc1O-proximal and a core lacO at the same position as Ptrc1O-core (Figure 3A). As the proximal lacO position of Ptrc and Ptrc1O-proximal has proven ineffective for repression and because PA1lacO-1 looks similar to Ptrc1O-core in its repression and induction pattern (Figure 5A), we agree with the previous study [28] that the core-located lacO appears to be causing the drop in expression. To test this, we characterized both Ptrc1O-core and PA1lacO-1 in the LacI-negative chloramphenicol resistance-only strain of Synechocystis. Indeed, both promoters were weaker than Ptrc1O-proximal, even when their activity without LacI is compared with the repressed activity of Ptrc1O-proximal (Figure 5A). Further, the activity of PA1lacO-1 was much lower than that of Ptrc1O-core in the absence of LacI, leading to a repression ratio of only 3.3 for PA1lacO-1 and 13 for Ptrc1O-core. It is possible that the slightly lower induction ratio for PA1lacO-1 as compared to the previous study [28] is due to lower levels of LacI/cell in this study. Finally, the reduced inherent promoter activity of Ptrc1O-core and PA1lacO-1 in Synechocystis as compared with E. coli further stresses our previous conclusion that tools to be used in a specific bacterium have to be functionally characterized in that particular chassis $[4,25]$ and illustrates the need for orthogonal and hence portable RNAP systems.

\section{Lacl-mediated DNA-looping in E. coli and Synechocystis}

To investigate the effect of tetrameric LacI-cooperativity on repression and DNA-looping in cyanobacteria, we designed a Ptrc-based library with two lacO to enable LacI-mediated DNA looping in analogy with previous designs for E. coli $[20,23,51]$. We added an auxiliary lacOsym at different distances, ranging from -2 to $21 \mathrm{bp}$, upstream of the Ptrc1O-proximal -35 element, while keeping the core Ptrc promoter and proximal lacOsym of Ptrc1Oproximal intact. As the Ptrc2O-library and Ptrc1O-proximal share identical promoters from the -35 box to the proximal lac operator and further produce identical 5' UTRs (Figures 3A and B), this enables us to directly compare them and detect effects from LacI-mediated DNA looping. Further, single lacOsym operators positioned upstream of a lacUV5 promoter -35 element have previously been shown to produce only minor effects in $E$. coli on expression in the presence of LacI and the absence of DNA-looping [51]. However, a subsequent study showed loop-independent effects of placing a single distal lacOsym too close to the promoter -35 element, especially for edge-to-edge distances between lacOsym and the -35 hexamer of 2 and 6 bp [20]. This effect was 
suggested to be due to interactions between LacI and the $E$. coli RNAP, or due to sequence-specific effects affecting the nearby promoter itself. Such sequence-specific effects are possible, and have been illustrated recently for cyanobacteria [5]. Therefore, the Ptrc2O-library can be compared with Ptrc1O-proximal to draw conclusions about the possible effects of DNA-looping, but caution should be taken in interpreting the data for the closest spacings of the -35 box to the distal lacOsym operator.

First of all, we can conclude that the repression and induction patterns of the Ptrc2O-library in E. coli (Figure 4B) and Synechocystis (Figure 5B) share many similarities. Both species produce a periodical repression and induction pattern with a period of $11 \mathrm{bp}$, illustrating that maximal repression can be achieved when both $l a c O$ are positioned on the same side of the DNA helix, and that minimal repression is produced when the operators are on opposite sides. To confirm that the maximal repression displayed in the troughs of the periodical pattern, and the minimal repression displayed in the peaks, are not due to promoter activity effects unrelated to LacI, we tested two highly repressed promoters and one weakly repressed promoter without LacI. Both the two highly repressed promoters were highly active in the absence of LacI, and the activity of the weakly repressed promoter was similar to the activity of the highly repressed promoters (Figure 5B), confirming that the periodical repression pattern is due to the presence of LacI. This confirms the numerous previous studies on LacI-mediated DNA looping in E. coli $[14,16-18,20,23,51]$, and demonstrates that DNA-looping as a mechanism of transcriptional regulation works in cyanobacteria, displaying the same number of average bp per turn of the supercoiled DNA helix as E. coli. This was anticipated, as structural DNA-binding proteins like HU that have been demonstrated to facilitate LacI-mediated DNA looping in E. coli [52] are present also in cyanobacteria (the Synechocystis HU gene is sll1712 in Cyanobase [53]). That a periodical repression pattern remains even under full IPTG induction in both E. coli and Synechocystis is not surprising. As stated previously, the binding of IPTG to LacI decreases the affinity of LacI to lacO approximately 1000 -fold [11]. Under circumstances when LacI is in excess to its operators, or its binding to a highaffinity operator is stabilized through DNA-looping, LacImediated DNA-loops could remain even under induced conditions. In fact, it has been shown that saturating induction does not completely remove loops in vivo [51] or in vitro [54]. To decrease the effect of residual repression even under full IPTG-induction, concentrations of LacI should not be higher than what is required for satisfactory repression. Further, just as in E. coli, the repression both for the non-induced and the induced conditions decrease with longer inter-lacO distances in Synechocystis. This has been shown before in $E$. coli and illustrates the drop in contribution to the local concentration of LacI at the primary proximal repression site from the distal site for longer distances $[23,55]$. On the other hand, it was recently demonstrated that the LacI-mediated DNA-loop itself is enough to repress transcription from a T7 promoter positioned inside the loop [55]. As this was demonstrated using the T7 RNAP, it is not tested whether bacterial RNAP will share the same sensitivity to DNA-loops, but likely, as the polymerases have evolved to perform the same function in similar ways. Hence, the decrease in repression for longer inter-lacO distances in Synechocystis may not only be due to a decreased local concentration of LacI at the primary repression site, but also due to a larger DNA-loop, which could exhibit a smaller hindrance for RNAP than a tighter one. Another similarity with $E$. coli is that the inter-lacO distances that represent maximum repression are not even multiples of $11 \mathrm{bp}$, corresponding to repression maxima at 55 and $66 \mathrm{bp}$, but one to two bp longer. This has been previously observed in vivo [23] and may be due to distortion of the intervening DNA sequence of the promoter by RNAP binding or the promoter DNA-sequence itself.

In Synechocystis, repression in the least optimal geometrical $l a c O$ positions, as represented by the expression peaks (Figure $5 \mathrm{~B}$ ), is very low even under non-induced conditions. As the Ptrc2O-library is identical to Ptrc1Oproximal, which is even less efficiently repressed, from the -35 element down to the TSS and the $5^{\prime}$ UTR, Ptrc2O-library promoters are expected to be similar to Ptrc1O-proximal in repression without DNA-looping. In fact, in the absence of LacI, the two highly repressed promoters $\mathrm{Ptrc} 2 \mathrm{O}-2$ and 13 were as or even more active than the fully induced Ptrc1O-prox (Figure 5A and B). Thus, Ptrc2O-library promoters whose $l a c O$ are out of phase are much less efficiently repressed in Synechocystis because of the weakly functioning proximal lac operator of Ptrc1O-proximal (Figure 5A and B), whereas their repression is stronger in E. coli for which Ptrc1O-proximal is more efficiently repressed (Figure $4 \mathrm{~A}$ and $\mathrm{B}$ ). As stated before, this difference in repression between $E$. coli NEB5 $\alpha$ and Synechocystis for Ptrc and Ptrc1O-prox could be due to the about five times lower numbers of $\mathrm{Lacl} /$ cell in the LacI-expressing Synechocystis strain. Thus, it is remarkable that repression of the Ptrc2Olibrary when the $l a c O$ are in phase is very efficient in Synechocystis, despite the weak primary proximal operator site and the lower levels of LacI/cell. In fact, the repression for geometrically optimal lacO positions is so efficient that IPTG induction almost fails to raise expression in Synechocystis, whereas it is possible to induce expression more in E. coli. Additionally, the repression troughs, which represent more optimal DNA-loop geometries, are wider in Synechocystis as compared to E. coli, and the expression peaks are narrower. Taken 
together, the stronger repression of an extremely leaky proximal lacOsym in optimal LacI-mediated DNA-loop configurations, the wider repression troughs, narrower expression peaks and the lower amounts of LacI/cell in Synechocystis as compared to E. coli have at least two possible implications. It implies that the Synechocystis RNAP is either more sensitive to DNA-looping than E. coli RNAP, and/or that Synechocystis chromatin DNA has lower in vivo torsion-resistance than $E$. coli chromatin DNA, lowering the barrier for forming twisted-DNA loops is the cyanobacterium.

\section{Conclusions}

Artificial and minimal promoters orthogonal to cyanobacteria can be used for stable constitutive expression and pose an alternative to the use of native promoters. The proximal lac operator site of Ptrc and its sibling Ptrc1Oproximal is insufficient for efficient repression of transcription in Synechocystis under the amounts of LacI/cell tested here. We show that in vivo DNA-looping works as a mechanism of transcriptional regulation in cyanobacteria similarly as in E. coli. DNA-looping appears to be an efficient mechanism of repression in cyanobacteria, as promoters carrying the inefficient proximal lacO site could be more strongly repressed in combination with auxiliary distal lac operators in optimal phases in Synechocystis than in E. coli. The weak repression of promoters with only the proximal lac operator site in Synechocystis could simply be due to insufficient numbers of LacI/cell and target operator. Yet, as some $\mathrm{Ptrc} 2 \mathrm{O}$ promoters are more efficiently repressed in Synechocystis than in E. coli despite the lower levels of Lac/cell in the cyanobacterium, we suggest that the repression differences could be due to previously observed differences in RNAP subunits. We tested for differences in the RNAP footprint using a proximal lacO promoter library but could not detect any discrepancy in pattern. The reasons for the more efficient repression of favorably-phased Ptrc $2 \mathrm{O}$ promoters in Synechocystis than in E. coli are unclear. We speculate that it could be due to differences in the ease with which the chromatin DNA is looped or twisted, or differences in RNAP sensitivity to deformed DNA. As DNA-looping is very efficient for LacI-repression of promoters in Synechocystis, we suggest that an optimal design should incorporate this mechanism. Further, to avoid unpredictable expression levels of different genes due to interactions between coding sequences and 5' UTRs on the mRNA level [56], future optimally repressed promoters would preferably involve DNA looping between core and distally located lacO. This allows for the exclusion of proximal lac operators from the 5' UTR and any possible effects they may have on the mRNA. Finally, the Ptrc2O (2) and (13) promoters display very high repression ratios in Synechocystis (about 400 and 170 times, respectively, Figure 5B), and could be used for genetic switches or gene expression systems that do not require subsequent induction. Alternatively, lower levels of LacI or unstable versions of LacI could be used to improve their inducibility.

\section{Methods}

\section{DNA parts and sequences}

All BioBrick parts, denoted by "BBa_", and BioBrick plasmids were obtained from the iGEM Registry of Standard Biological Parts [34] (referred to as the iGEM Registry). Colony PCR on BioBrick plasmids was performed using the VF2 (BBa_G00100) and VR (BBa_G00101) flanking primers. Primer sequences (Table 2), promoter sequences (Table 1) and construct sequences (Additional file 1) are available.

\section{Strains and culture conditions}

Escherichia coli strain NEB5 $\alpha$ F' $I^{q}\left(\right.$ lac $^{q}$ strain, New England Biolabs, referred to as E. coli) was used for cloning and promoter characterization of the constitutive, control and Ptrc2O-library promoters. E. coli strain DH5 $\alpha Z 1$ (carries two lacl $^{q}$ genes at the attB locus, Expressys) was used for cloning and promoter characterization of the proximal $l a c O$-library. The $l a c l^{q}$ gene is a promoter-mutated version of the wild-type lacI gene that causes about ten times as much LacI to be expressed as compared with the wild-type strain [57]. The strains were cultured in $\mathrm{LB}$ medium at $37^{\circ} \mathrm{C}$ shaking at $225 \mathrm{rpm}$, or on LB-agar plates, and were supplemented with $25 \mu \mathrm{g} / \mathrm{mL}$ kanamycin for pPMQAK1 [25] [iGEM Registry: BBa_J15 3000, Genbank: GU933126, Addgene: 26052] constructs, $50 \mu \mathrm{g} / \mathrm{mL}$ kanamycin for pSB1AK3 [34] constructs or $35 \mu \mathrm{g} / \mathrm{mL}$ chloramphenicol for the Synechocystis lacI or Chloramphenicol resistance-only recombination vectors.

Synechocystis PCC 6803 (the glucose tolerant strain ATCC 27184, referred to as Synechocystis) was cultured in BG11 medium [58], or on BG11-agar plates, supplemented with $50 \mu \mathrm{g} / \mathrm{mL}$ kanamycin for pPMQAK1 constructs. The Synechocystis engineered strain expressing LacI and the strain carrying only Chloramphenicol resistance were cultured in BG11 supplemented with $25 \mu \mathrm{g} / \mathrm{mL}$ chloramphenicol, or both $25 \mu \mathrm{g} / \mathrm{mL}$ kanamycin and $25 \mu \mathrm{g} / \mathrm{mL}$ chloramphenicol when containing pPMQAK1 constructs. All strains were grown at $30^{\circ} \mathrm{C}$ under continuous white light of $20-50 \mu \mathrm{mol}$ photons $\mathrm{m}^{-2} \mathrm{~s}^{-1}$ shaking at $120 \mathrm{rpm}$ unless otherwise stated.

\section{Construction and characterization of constitutive promoters in Synechocystis}

Our selection criteria for promoters expressing the Lac repressor in Synechocystis were minimal constitutive promoters orthogonal to Synechocystis. For this purpose, we selected several members of an artificial $\sigma-70$ promoter 
Table 2 Primer DNA sequences

\begin{tabular}{|c|c|}
\hline Primer name & Sequence $5^{\prime}$ to $3^{\prime}$ \\
\hline VF2 (BBa_G00100) & TGCCACCTGACGTCTAAGAA \\
\hline VR (BBa_G00101) & ATTACCGCCTTTGAGTGAGC \\
\hline RBS*-EYFP-BB-f1 & CTTCTAGAGTAGTGGAGGTTACTAGATGGTGAGCAAGGGCG \\
\hline B0015-BB-r1 & GAACTGCAGCGGCCGCTACTAGTATATAAACGCAGAAAGGCCC \\
\hline j5_00029 & GCGGCCGCTGCAGCCCGTAGAAAAGATCAAAGGATCTTCTTGAGA \\
\hline j5_00030 & GCCACGTAGGGGTCTCTAGAAGCGGCCGCGAATTCATGTGAGCAAAAGGCCAGCA \\
\hline j5_00031 & GCCGCTTCTAGAGACCCCTACGTGGCCGGCAATGGTCC \\
\hline j5_00032 & GGTGAAACTGACCGAACATAGGAGACTTGGTGGGCTGGCCG \\
\hline j5_00033 & CCACCAAAGTCTCCTATGTTCGGTCAGTTTCACCTGATTTACG \\
\hline j5_00079 & $\begin{array}{l}\text { CTGGTTTCACATTCACCATGATCAAACCTCCACTACTCTAGTAGCTAGCATTGTACCTAGGACTG } \\
\text { AGCTAGCCATAAAGACAGTCATTCATCTITCTGCCCCTCC }\end{array}$ \\
\hline j5_00042 & GTAGTGGAGGTTTGATCATGGTGAATGTGAAACCAGTAACGTTATACGATG \\
\hline j5_00072 & GTTCGTTAAGGCTTGATCTCTATTATTACTGCCCGCTTTCCAGTCGG \\
\hline j5_00073 & GGAAAGCGGGCAGTAATAATAGAGATCAAGCCTTAACGAACTAAGACCCC \\
\hline j5_00088 & CCCGATCAACTCGTGTCTGCTCCTCGGTTATGTTITTAAGGTC \\
\hline j5_00090 & CGAGGAGCAGACACGAGTTGATCGGGCACGTAAGAGG \\
\hline j5_00092 & GCCATCCACTTCCTTGGTCTGACAGCTCGAGGCTTGG \\
\hline j5_00093 & GCTGTCAGACCAAGGAAGTGGATGGCCCCGTATTGC \\
\hline j5_00039 & CTITGATCTITTCTACGGGCTGCAGCGGCCGCTACTAGTAGGTCCCAAGTTTGTGCTGTGGC \\
\hline slr0168-UR-f2 & AAAGGGGACGAAGCCGCAGTTC \\
\hline slr0168-DR-r2 & ATCGCCGTGGTTAAATCCGTGG \\
\hline P2OLib-r & TGAGCTACTAGTATGTGTGAAATTGTGAGCGCTCACAATTCCAC \\
\hline P2OLib-f1O & TCAGAATTCGCGGCCGCTTCTAGAGCGAACGGTTCTGGCAAATATTC \\
\hline P2OLib-f-2 & TCAGAATTCGCGGCCGCTTCTAGAGAATTGTGAGCGCTCACAATTGACAATTAATCATCCGGCTCG \\
\hline P2OLib-f-1 & TCAGAATTCGCGGCCGCTTCTAGAGAATTGTGAGCGCTCACAATTTGACAATTAATCATCCGGCTCG \\
\hline P2OLib-f0 & TCAGAATTCGCGGCCGCTTCTAGAGAATTGTGAGCGCTCACAATTTTGACAATTAATCATCCGGCTC \\
\hline P2OLib-f1 & TCAGAATTCGCGGCCGCTTCTAGAGAATTGTGAGCGCTCACAATTGTTGACAATTAATCATCCGGC \\
\hline P2OLib-f2 & TCAGAATTCGCGGCCGCTTCTAGAGAATTGTGAGCGCTCACAATTTGTTGACAATTAATCATCCGG \\
\hline P2OLib-f3 & TCAGAATTCGCGGCCGCTTCTAGAGAATTGTGAGCGCTCACAATTCTGTTGACAATTAATCATCCGG \\
\hline P2OLib-f4 & TCAGAATTCGCGGCCGCTTCTAGAGAATTGTGAGCGCTCACAATTGCTGTTGACAATTAATCATCCGG \\
\hline P2OLib-f5 & TCAGAATTCGCGGCCGCTTCTAGAGAATTGTGAGCGCTCACAATTAGCTGTTGACAATTAATCATCCG \\
\hline P2OLib-f6 & TCAGAATTCGCGGCCGCTTCTAGAGAATTGTGAGCGCTCACAATTGAGCTGTTGACAATTAATCATCCG \\
\hline P2OLib-f7 & TCAGAATTCGCGGCCGCTTCTAGAGAATTGTGAGCGCTCACAATTTGAGCTGTTGACAATTAATCATCC \\
\hline P2OLib-f8 & TCAGAATTCGCGGCCGCTTCTAGAGAATTGTGAGCGCTCACAATTATGAGCTGTTGACAATTAATCATCC \\
\hline P2OLib-f9 & TCAGAATTCGCGGCCGCTTCTAGAGAATTGTGAGCGCTCACAATTAATGAGCTGTTGACAATTAATCATCC \\
\hline P2OLib-f10 & TCAGAATTCGCGGCCGCTTCTAGAGAATTGTGAGCGCTCACAATTAAATGAGCTGTTGACAATTAATCATC \\
\hline P2OLib-f11 & TCAGAATTCGCGGCCGCTTCTAGAGAATTGTGAGCGCTCACAATTGAAATGAGCTGTTGACAATTAATCATC \\
\hline P2OLib-f12 & TCAGAATTCGCGGCCGCTTCTAGAGAATTGTGAGCGCTCACAATTTGAAATGAGCTGTTGACAATTAATC \\
\hline P2OLib-f13 & TCAGAATTCGCGGCCGCTTCTAGAGAATTGTGAGCGCTCACAATTCTGAAATGAGCTGTTGACAATTAATC \\
\hline P2OLib-f14 & TCAGAATTCGCGGCCGCTTCTAGAGAATTGTGAGCGCTCACAATTTCTGAAATGAGCTGTTGACAATTAATC \\
\hline P2OLib-f15 & TCAGAATTCGCGGCCGCTTCTAGAGAATTGTGAGCGCTCACAATTTCTGAAATGAGCTGTTGACAATTA \\
\hline P2OLib-f16 & TCAGAATTCGCGGCCGCTTCTAGAGAATTGTGAGCGCTCACAATTATTCTGAAATGAGCTGTTGACAATT \\
\hline P2OLib-f17 & TCAGAATTCGCGGCCGCTTCTAGAGAATTGTGAGCGCTCACAATTTATTCTGAAATGAGCTGTTGACAATT \\
\hline P2OLib-f18 & TCAGAATTCGCGGCCGCTTCTAGAGAATTGTGAGCGCTCACAATTATATTCTGAAATGAGCTGTTGACAATT \\
\hline
\end{tabular}


Table 2 Primer DNA sequences (Continued)

\begin{tabular}{|c|c|}
\hline P2OLib-f19 & TCAGAATTCGCGGCCGCTTCTAGAGAATTGTGAGCGCTCACAATTAATATTCTGAAATGAGCTGTTGACAA \\
\hline P2OLib-f20 & TCAGAATTCGCGGCCGCTTCTAGAGAATTGTGAGCGCTCACAATTAAATATTCTGAAATGAGCTGTTGACA \\
\hline P2OLib-f21 & TCAGAATTCGCGGCCGCTTCTAGAGAATTGTGAGCGCTCACAATTCAAATATTCTGAAATGAGCTGTTGAC \\
\hline P1Od-f1 & $\begin{array}{l}\text { AAGTCTAGAGAATTGTGAGCGCTCACAATTITGACAATTAATCATCCGGCTCGTATAATGTGTGGAATCAC } \\
\text { ACATACTAGAGTAGTGGAGGTTACTAGATGG }\end{array}$ \\
\hline P1Oc-f1 & $\begin{array}{l}\text { AAGTCTAGAGAAATATTCTGAAATGAGCTGTTGACAATTGTGAGCGCTCACAATATAATGTGTGGAATCAC } \\
\text { ACATACTAGAGTAGTGGAGGTTACTAGATGG }\end{array}$ \\
\hline PA1lacO1-FP-f1 & $\begin{array}{l}\text { AAGTCTAGAGAAAGAGTGTTGACTTGTGAGCGGATAACAATGATACTTAGATTCAATTGTGAGCGGATAAC } \\
\text { AATTTCACACATACTAGAGTAGTGGAGGTTACTAGATGG }\end{array}$ \\
\hline PtrcOs-prox-8-f1 & $\begin{array}{l}\text { TTCTCTAGAGTTGACAATTAATCATCCGGCTCGTATAATGTGTAATTGTGAGCGCTCACAATTTACTAGAGTA } \\
\text { GTGGAGGTTACTAGATGG }\end{array}$ \\
\hline PtrcOs-prox-9-f1 & $\begin{array}{l}\text { TTCTCTAGAGTTGACAATTAATCATCCGGCTCGTATAATGTGTGAATTGTGAGCGCTCACAATTTACTAGAG } \\
\text { TAGTGGAGGTTACTAGATGG }\end{array}$ \\
\hline PtrcOs-prox-10-f1 & $\begin{array}{l}\text { TTCTCTAGAGTTGACAATTAATCATCCGGCTCGTATAATGTGTGGAATTGTGAGCGCTCACAATTTACTAGA } \\
\text { GTAGTGGAGGTTACTAGATGG }\end{array}$ \\
\hline PtrcOs-prox-11-f1 & $\begin{array}{l}\text { TTCTCTAGAGTTGACAATTAATCATCCGGCTCGTATAATGTGTGGAAATTGTGAGCGCTCACAATTTACTAG } \\
\text { AGTAGTGGAGGTTACTAGATGG }\end{array}$ \\
\hline PtrcOs-prox-12-f1 & $\begin{array}{l}\text { TTCTCTAGAGTTGACAATTAATCATCCGGCTCGTATAATGTGTGGAGAATTGTGAGCGCTCACAATTTACTA } \\
\text { GAGTAGTGGAGGTTACTAGATGG }\end{array}$ \\
\hline PtrcOs-prox-13-f1 & $\begin{array}{l}\text { TTCTCTAGAGTTGACAATTAATCATCCGGCTCGTATAATGTGTGGAGTAATTGTGAGCGCTCACAATTTACT } \\
\text { AGAGTAGTGGAGGTIACTAGATGG }\end{array}$ \\
\hline PtrcOs-prox-14-f1 & $\begin{array}{l}\text { TTCTCTAGAGTTGACAATTAATCATCCGGCTCGTATAATGTGTGGAGTCAATTGTGAGCGCTCACAATTTAC } \\
\text { TAGAGTAGTGGAGGTTACTAGATGG }\end{array}$ \\
\hline PtrcOs-prox-15-f1 & $\begin{array}{l}\text { TTCTCTAGAGTTGACAATTAATCATCCGGCTCGTATAATGTGTGGAGTCGAATTGTGAGCGCTCACAATTA } \\
\text { CTAGAGTAGTGGAGGTTACTAGATGG }\end{array}$ \\
\hline PtrcOs-prox-16-f1 & $\begin{array}{l}\text { TTCTCTAGAGTTGACAATTAATCATCCGGCTCGTATAATGTGTGGAGTCGTAATTGTGAGCGCTCACAATTT } \\
\text { ACTAGAGTAGTGGAGGTTACTAGATGG }\end{array}$ \\
\hline PtrcOs-prox-17-f1 & $\begin{array}{l}\text { TTCTCTAGAGTTGACAATTAATCATCCGGCTCGTATAATGTGTGGAGTCGTCAATTGTGAGCGCTCACAATTा } \\
\text { ACTAGAGTAGTGGAGGTTACTAGATGG }\end{array}$ \\
\hline PtrcOs-prox-18-f1 & $\begin{array}{l}\text { TTCTCTAGAGTTGACAATTAATCATCCGGCTCGTATAATGTGTGGAGTCGTCCAATTGTGAGCGCTCACAAT } \\
\text { TIACTAGAGTAGTGGAGGTTACTAGATGG }\end{array}$ \\
\hline PtrcOs-prox-20-f1 & $\begin{array}{l}\text { TTCTCTAGAGTTGACAATTAATCATCCGGCTCGTATAATGTGTGGAGTCGTCCAGAATTGTGAGCGCTCACA } \\
\text { ATTACTAGAGTAGTGGAGGTTACTAGATGG }\end{array}$ \\
\hline PtrcOs-prox-22-f1 & $\begin{array}{l}\text { TTCTCTAGAGTTGACAATTAATCATCCGGCTCGTATAATGTGTGGAGTCGTCCAGACAATTGTGAGCGCTCA } \\
\text { CAATTIACTAGAGTAGTGGAGGTTACTAGATGG }\end{array}$ \\
\hline PtrcOs-prox-24-f1 & $\begin{array}{l}\text { TTCTCTAGAGTTGACAATTAATCATCCGGCTCGTATAATGTGTGGAGTCGTCCAGACTCAATTGTGAGCGCT } \\
\text { CACAATTTACTAGAGTAGTGGAGGTTACTAGATGG }\end{array}$ \\
\hline
\end{tabular}

library spanning a wide range of activities in E. coli for characterization in Synechocystis (BBa_J23100, BBa_J23101, $\mathrm{BBa} \_$23102, BBa_J23105, BBa_J23106, BBa_J23109, BBa J23113 and BBa_J23114, from the iGEM Registry's Anderson collection [34]). For comparison, we selected three promoters commonly used for engineered expression in Synechocystis; the nitrate reductase promoter PnirA (synthesized by Epoch Biolabs Inc.), the plastocyanin promoter PpetE (BBa_K273019, from iGEM-team UppsalaSweden 2009) and the RNase P subunit B promoter PrnpB [25]. An EYFP reporter cassette containing $\mathrm{RBS}^{*}$, a ribosome binding site that is highly active in both $E$. coli and Synechocystis [4], EYFP itself (BBa_E0030) and a double transcriptional terminator (BBa_B0015) was constructed by PCR using the Phusion Hot-Start II High-Fidelity DNA polymerase (Thermo Scientific, referred to as Phusion) with primers RBS*-EYFP-BB-f1, B0015-BB-r1 and another EYFP reporter cassette as template (BBa_E0430) according to the manufacturer's instructions. The promoters were cloned together with the EYFP reporter cassette into pSB1AK3 using BioBrick 3A-assembly [59], subcloned into pPMQAK1 and confirmed by sequencing. The pPM QAK1-carried constructs plus an empty pPMQAK1 vector control plasmid [25] were transferred to Synechocystis through conjugation according to a previously published procedure [4] using E. coli strain HB101 with pRL443 [60] 
as the conjugal plasmid and BG11 plates with $50 \mu \mathrm{g} / \mathrm{mL}$ kanamycin for selection. Single Synechocystis colonies were grown in flat-bottomed tissue culture 6-well plates (Sarstedt) with lids taped shut with Micropore tape in $5 \mathrm{~mL}$ BG11 supplemented with $50 \mu \mathrm{g} / \mathrm{mL}$ kanamycin at ca $50 \mu \mathrm{mol}$ photons $\mathrm{m}^{-2} \mathrm{~s}^{-1}$ (protecting cultures from light with white paper the first days) for about two weeks (corresponding to a final absorbance at $750 \mathrm{~nm}$ (Abs750) of 0.6-1.3 as measured in translucent 96-well tissue culture plates using a Chameleon V Microplate Reader (Hidex, referred to as plate reader)). The presence of the promoter-reporter plasmids was confirmed with colony PCR and the cultures were inspected for the absence of $E$. coli using microscopy. To produce promoter characterization seed cultures in the same growth phase, all cultures were diluted to Abs750 = 0.01 into E-flasks with $25 \mathrm{~mL}$ medium and grown for four days in the same conditions as before, reaching a final Abs750 of 0.3-0.45. The seed cultures were used to inoculate measurement cultures to Abs750 $=0.01$, which were split into six replicate cultures each of $5 \mathrm{~mL}$ in 6 -well plates with sealed lids. The measurement cultures were grown for 42 hours whereupon samples were taken for plate reader measurements of Abs750 and EYFP fluorescence using $485 \mathrm{~nm}$ emission and $535 \mathrm{~nm}$ excitation filters. Black 96-well plates with clear flat bottom (BD Falcon) were used to eliminate well-to-well fluorescence cross-talk. After subtracting medium and instrument background, fluorescence per cell values were calculated by normalizing fluorescence values with Abs750 values, averaging over the six replicates and estimating the standard error of the mean. Finally, specific fluorescence per cell values for each promoter construct was obtained by subtracting cellular background fluorescence from the empty pPMQAK1 control culture.

\section{Construction of Lacl-expressing and Chloramphenicol resistance-only strains of Synechocystis}

As we have previously found that the PrnpB promoter in combination with the BBa_B0034 RBS express amounts of LacI strongly repressing $\mathrm{Ptrc} 2 \mathrm{O}$ when both constructs are carried on the pPMQAK1 plasmid [25], we selected the weaker (as characterized in this study) and synthetic BBa_J23114 promoter in combination with RBS* [4] to express LacI from the chromosome. For insertion of the LacI-expressing cassette into the chromosome we selected slr0168, a previously used neutral site $[61,62]$ that displays very low transcript levels [37]. The LacI-expression cassette, which is flanked by slr0168 insertion sequences, consists of a forward terminator to insulate the cassette from potentially incoming chromosomal RNAP ( $r$ pB_T1 from E. coli), BBa_J23114 in combination with $\mathrm{RBS}^{*}$ to drive LacI expression, the lacI CDS, another forward terminator (ilvGEDA_T from E. coli) and a chloramphenicol resistance cassette $(\mathrm{CmR})$ derived from pSB1AC3 (iGEM Registry). The LacI-expression cassette together with a pMB1 replicon for replication in $E$. coli were assembled using one-step isothermal assembly [63] using the j5 DNA assembly design automation software [64] for primer design. Specifically, primers j5_00029 and j5_00030 were used to PCR the pMB1 replicon using pBluescript II SK + (Stratagene) as template, j5_00031 and j5_00032 to PCR the slr0168 upstream recombination recombination sequence using Synechocystis genomic DNA as template, j5_00033 and j5_00079 to PCR rnpB_T1 and the promoter-RBS region using $r n p B \_\mathrm{T} 1$ synthetic DNA (Genscript) as template, j5_00042 and j5_00072 to PCR lacI using E. coli strain JM107 genomic DNA as template, j5_00073 and j5_00088 to PCR ilvGEDA_T using synthetic DNA (Genscript) as template, j5_00090 and j5_00092 to PCR CmR using pSB1AC3 as template and finally j5_00093 and j5_00039 to PCR the slr0168 downstream recombination sequence using Synechocystis genomic DNA as template. The upstream recombination and the rnpB_T1-promoter-RBS fragments, and the ilvGE$D A_{-} \mathrm{T}$ and $\mathrm{CmR}$ fragments, were spliced together by overlap extension (SOE) before one-step isothermal assembly. The chloramphenicol resistance-only construct was constructed analogously to the LacI-expressing construct, but all elements except the recombination sites, the second forward terminator (ilvGEDA_T) and the chloramphenicol resistance cassette were excluded. The sequences of both constructs are available in Additional file 1. All PCR and SOE reactions were made using Phusion. The resulting pMB1-replicated LacI-expression cassette and the chloramphenicol resistance-only construct were sequenceconfirmed, transferred to Synechocystis by natural transformation and inserted into the genomic slr0168 target site by homologous recombination according to previously published procedures [4]. Mutants were selected on BG11 plates supplemented with $10 \mu \mathrm{g} / \mathrm{mL}$ chloramphenicol and single colonies were streaked on BG11 $25 \mu \mathrm{g} / \mathrm{mL}$ chloramphenicol plates twice to allow for full segregation. Finally, the fully segregated mutants were confirmed with colony PCR and sequencing of the insertion site using primers slr0168-UR-f2 and slr0168-DR-r2.

To confirm and quantify the expression of LacI in the LacI-expressing strain of Synechocystis, a quantitative western blot was performed with anti-LacI polyclonal antibodies (PAB10255, raised in rabbit, Abnova) and secondary HRP-conjugated anti-rabbit antibodies (ImmunStar GAR-HRP conjugate, \#170-5046, BioRad). Briefly, a known number of cells from three biological replicates of DH5 $\alpha$ Z1, two replicates of NEB5 $\alpha$, and three replicates of the LacI-expressing strain of Synechocystis carrying different promoter reporter constructs on PPMQAK1, were denatured in reducing SDS sample buffer for 5 mins at $95^{\circ} \mathrm{C}$. The samples were run on a PAGE gel (any-kD 
precast 8-well gels, BioRad) and blotted to membranes (TransBlot turbo, midi transfer packs with PVDF membranes, BioRad). Western blotting was performed according to the manufacturer's instruction with the exception that the primary LacI antibody was hybridized over night. The blot was developed using HRP chemiluminescence (Immun-Star HRP substrate kit, BioRad) and the band intensities were quantified using QuantityOne software (BioRad). The average band intensities were normalized with the number of loaded cells to get relative values of LacI/cell for the different strains. For an absolute value of LacI tetramers/cell for all strains, a previous quantification of 3000 tetramers of LacI/cell for DH5 $\alpha$ Z1 [36] was used as a standard.

\section{Construction and characterization of the Ptrc-derived promoter library in E. coli and Synechocystis}

The Ptrc2O-library and Ptrc1O-prox promoters were made with Phusion PCR using the reverse primer P2OLib-r to exchange lacO1 of the original Ptrc [24] and Ptrc2O [25] (here referred to as Ptrc2O-orig) to the symmetric lacOsym operator. Different forward primers adding another lacOsym upstream the -35 box with spacers of different length in between (primers P2OLib-f-n for the Ptrc2O-library, where $\mathrm{n}$ is a numeral representing the spacer length ranging from -2 to 21 , and primer P2OLib-f1O for Ptrc1O-prox that does not include a second lacOsym) were used, together with Ptrc2O-orig as PCR template. The promoter PCR products were assembled with the same EYFP reporter cassette as the constitutive promoters into pPMQAK1 using 3A-assembly. The single lacOsym control promoters Ptrc1O-dist, with lacOsym immediately distal to the -35 box, and Ptrc1O-core, with a slightly truncated lacOsym in the core region between the -35 and the -10 boxes, and the PA1lacO-1 promoter [36], were made with Phusion PCR using the different forward primers P1Od-f1 (for Ptrc1O-dist), P1Oc-f1 (for Ptrc1Ocore) and PA1lacO1-FP-f1 (for PA1lacO-1) and the same B0015-BB-r1 reverse primer using the EYFP reporter cassette as template. These PCR products were also cloned into pPMQAK1 using the BioBrick cloning site. After sequence confirmation using the VF2 and VR primers all promoters were first characterized in $E$. coli and then transferred to the LacI-expressing Synechocystis strain for subsequent characterization.

For characterization of the Ptrc-derived promoter library in $E$. coli the following protocol was repeated at three different days, producing a total of six biological measurement replicates. Two overnight cultures for each promoter construct were inoculated from frozen glycerol stocks in $2 \mathrm{~mL}$ LB supplemented with $25 \mu \mathrm{g} / \mathrm{mL}$ kanamycin in $13 \mathrm{~mL}$ growth tubes (Sarstedt) and grown at $37^{\circ} \mathrm{C}$ shaking at $225 \mathrm{rpm}$ until all cultures had reached stationary phase (approximately 22 hours). The overnight cultures were put into ice-water to ensure that both the negative and positive Isopropyl $\beta$-D-1-thiogalactopyranoside (IPTG) cultures had the same starting conditions. For each culture, $1 \mu \mathrm{l}$ was transferred to a 96-well tissue culture plate pre-loaded with $99 \mu \mathrm{LB}$ supplemented with $25 \mu \mathrm{g} / \mathrm{mL}$ kanamycin and either 0 or $1 \mathrm{mM}$ IPTG for the non-induced plate and induced plate, respectively. Only the middle 60 wells were used as the evaporation rate was observed to be higher for the outermost wells. The plates were sealed with Breathe-Easy sealing membranes (SigmaAldrich) and incubated shaking at level 8 on a Delfia plate shaker (Wallac) at $37^{\circ} \mathrm{C}$ for 7 hours. Samples were taken and measured in black 96-well plates with clear flat bottom in the same way as for the constitutive promoters in Synechocystis, but Abs595 was used instead of Abs750.

For characterization in Synechocystis the Ptrc-derived promoter library was transferred to the LacI-expressing Synechocystis strain by conjugation as was done for the constitutive promoters to wild-type Synechocystis, but BG11 plates with $25 \mu \mathrm{g} / \mathrm{mL}$ kanamycin and $25 \mu \mathrm{g} / \mathrm{mL}$ chloramphenicol was used for selection. Two colonies for each promoter construct were inoculated into ca $2 \mathrm{~mL}$ BG11 with $25 \mu \mathrm{g} / \mathrm{mL}$ kanamycin and $25 \mu \mathrm{g} / \mathrm{mL}$ chloramphenicol in $13 \mathrm{~mL}$ growth tubes shaking in low light conditions (ca $5 \mu \mathrm{mol}$ photons $\mathrm{m}^{-2} \mathrm{~s}^{-1}$ ) for ca three weeks (reaching a final Abs750 ranging from 1 to 3.5). To ensure that the seed cultures are in the same growth phase all cultures were diluted to Abs750 $=0.05$ and grown under the same conditions as the parent cultures for 44 hours. For measurements, all cultures were diluted once more in the $13 \mathrm{~mL}$ growth tubes to Abs750 $=0.05$ and $200 \mu \mathrm{l}$ of each was loaded into the middle 32 wells of 96 -well tissue culture plates, where the induction plates were pre-loaded with $1 \mu \mathrm{l} 200 \mathrm{mM}$ IPTG, and mixed by pipetting. The plate measurement cultures were grown with the lids on shaking at level 4 on a Delfia plateshaker at $30^{\circ} \mathrm{C}$ in white light of ca $50 \mu \mathrm{mol}$ photons $\mathrm{m}^{-2} \mathrm{~s}^{-1}$ for 46 hours, after which samples were taken for measurements. The cultures in the $13 \mathrm{~mL}$ growth tubes were put back in the same low-light conditions immediately after starting the first measurement cultures and after two days they were used to start new measurement cultures, leading to a total of four biological replicates for all promoter constructs. The second round of measurement cultures were produced in the same way as the first one, but the cultures were diluted to Abs750=0.05 directly in the 96-well plates, and grown under the same conditions for the same time. Samples were taken and measured in black 96-well plates with clear flat bottom in the same way as for the constitutive promoters in Synechocystis, but averages and standard errors of the mean were calculated for four replicates instead of six.

The control promoter constructs BBa_J23101, Ptrc1Ocore, PA1lacO-1 and the Ptrc2O-library constructs Ptrc2O- 
2, 13 and 18 were transferred into the chloramphenicol resistance-only strain of Synechocystis to enable characterization of these promoters without the presence of LacI. Conjugation and characterization was carried out in a similar way as for the LacI-expressing Synechocystis strain, but the measurement cultures were inoculated into $1 \mathrm{~mL}$ medium in $13 \mathrm{~mL}$ growth tubes instead of 96-well plates and grown at 10-15 $\mu \mathrm{mol}$ photons $\mathrm{m}^{-2} \mathrm{~s}^{-1}$. Only one round of measurement cultures were produced (yielding two biological replicates). The cultures were measured in the same way as for the LacI-expressing Synechocystis strain but averages and standard errors of the mean were calculated for two replicates instead of four.

\section{Construction and characterization of the proximal lacO-library in E. coli DH5aZ1 and Synechocystis}

The proximal $l a c O$ promoter library was made with Phusion PCR using reverse primer B0015-BB-r1 together with the forward primers PtrcOs-prox- $n-f 1$. The letter $n$ is a numeral ranging from 8-18 in steps of one and from 18-24 in steps of two, representing the distance in bp between the center of a proximal lacOsym to the TSS of a Ptrc core promoter (Table 1). The PCR was performed using a strongly enhanced green fluorescent protein (SGFP2) [65] codon-optimized for E. coli (DNA 2.0, a gift from Erik Gullberg) reporter construct consisting of RBS*-SGFP2-BBa_B0015 (Additional file 1) as template. The PCR products were cloned into PPMQAK1 using the BioBrick cloning site. After sequence confirmation using the VF2 and VR primers all promoters were characterized in E. coli DH5 $\alpha \mathrm{Z} 1$ and transferred to both the LacIexpressing Synechocystis strain and the chloramphenicol resistance-only strain for subsequent characterization.

Characterization in E. coli DH5 $\alpha \mathrm{Z} 1$ was done similarly as for the Ptrc-derived promoter library in NEB5 $\alpha$ but six biological replicates were characterized divided on two different days. The cultures were grown directly in black 96-well plates with clear flat bottom diluted 1:200 in $200 \mu \mathrm{l}$ medium supplemented with water or $2 \mathrm{mM}$ IPTG, shaking at level 7 on the plate shaker. The cultures were measured without lids at several time-points after the start of the experiment (approximately 0, 2.4, $3.8,4.2,4.7,5.1$ and 5.5 hours after dilution and induction) to observe for possible fluorescence/absorbance steady states and the temporal development of the induction ratios. The induction ratios and their estimated standard errors were calculated from the ratio of induced/non-induced fluorescence/absorbance data.

Conjugation to and characterization in the LacI-expressing Synechocystis strain and the chloramphenicol resistance-only strain was done similarly as for the Ptrc-derived promoter library in the LacI-expressing Synechocystis strain. The measurement cultures were inoculated into $1 \mathrm{~mL}$ medium supplemented with water or $2 \mathrm{mM}$ IPTG in
$13 \mathrm{~mL}$ growth tubes instead of 96-well plates, and grown at $10-15 \mu \mathrm{mol}$ photons $\mathrm{m}^{-2} \mathrm{~s}^{-1}$. Only one round of measurement cultures were produced (yielding two biological replicates). The cultures were measured in the same way as the Ptrc-derived promoter library in the LacI-expressing Synechocystis strain. The induction ratios and their estimated standard errors were calculated from the ratio of induced/non-induced fluorescence/absorbance data for the Lacl-expressing Synechocystis strain and the repression ratios and their estimated standard errors were calculated from the ratio of the fluorescence/absorbance data of the chloramphenicol resistance-only strain/the data from the LacI-expressing strain.

\section{Additional file}

Additional file 1: DNA sequences of the EYFP reporter, the SGFP2 reporter, the Lacl-expression cassette and the chloramphenicol resistance-only construct.

\section{Competing interests}

The authors declare that they have no competing interests.

\section{Author's contributions}

DC, TH and PL conceived of the research; DC designed the study and performed the experiments; DC and PL analyzed the data; DC and PL wrote the manuscript. All authors approved of the final manuscript.

\section{Acknowledgements}

We would like to acknowledge financial support from the Swedish Energy Agency; the Knut and Alice Wallenberg Foundation (project MoSE); and the European Union Seventh Framework Programme (FP7/2007-2013) under grant agreement number 308518 (CyanoFactory).

\section{Author details}

${ }^{1}$ Microbial Chemistry, Department of Chemistry-Ångström Laboratory, Science for Life Laboratory, Uppsala University, P.O. Box 523, SE-75120 Uppsala, Sweden. ${ }^{2}$ Bioforsk, Frederik A Dahls vei 20, 1432 Ås Oslo, Norway.

Received: 16 August 2013 Accepted: 26 December 2013 Published: 27 January 2014

\section{References}

1. Ducat DC, Way JC, Silver PA: Engineering cyanobacteria to generate high-value products. Trends Biotechnol 2011, 29:95-103.

2. Lindblad P, Lindberg P, Oliveira P, Stensjö K, Heidorn T: Design, engineering, and construction of photosynthetic microbial cell factories for renewable solar fuel production. Ambio 2012, 41:163-168.

3. Keasling JD: Synthetic biology and the development of tools for metabolic engineering. Metab Eng 2012, 14:189-195.

4. Heidorn T, Camsund D, Huang HH, Lindberg P, Oliveira P, Stensjö K, Lindblad P: Synthetic biology in cyanobacteria engineering and analyzing novel functions. Methods Enzymol 2011, 497:539-579.

5. Huang HH, Lindblad P: Wide-dynamic-range promoters engineered for cyanobacteria. J Biol Eng 2013, 7:10.

6. Cardinale S, Arkin AP: Contextualizing context for synthetic biology identifying causes of failure of synthetic biological systems. Biotechnol J 2012, 7:856-866.

7. Rao CV: Expanding the synthetic biology toolbox: engineering orthogonal regulators of gene expression. Curr Opin Biotechnol 2012 23:689-694.

8. Wang B, Wang J, Zhang W, Meldrum DR: Application of synthetic biology in cyanobacteria and algae. Front Microbiol 2012, 3:344.

9. Gilbert W, Müller-Hill B: Isolation of the lac repressor. Proc Natl Acad Sci USA 1966, 56:1891-1898. 
10. Lewis M: The lac repressor. C R Biol 2005, 328:521-548.

11. Barkley $M D$, Riggs $A D$, Jobe $A$, Burgeois $S$ : Interaction of effecting ligands with lac repressor and repressor-operator complex. Biochemistry (Mosc) 1975, 14:1700-1712.

12. Sadler JR, Sasmor $H$, Betz JL: A perfectly symmetric lac operator binds the lac repressor very tightly. Proc Natl Acad Sci U S A 1983, 80:6785-6789.

13. Oehler S, Amouyal M, Kolkhof P, Von Wilcken-Bergmann B, Muller-Hill B: Quality and position of the three lac operators of $E$. coli define efficiency of repression. EMBO J 1994, 13:3348-3355.

14. Mossing MC, Record MT Jr: Upstream operators enhance repression of the lac promoter. Science 1986, 233:889-892.

15. Krämer $H$, Niemoller $M$, Amouyal $M$, Revet B, Von Wilcken-Bergmann B, Muller-Hill B: lac repressor forms loops with linear DNA carrying two suitably spaced lac operators. EMBO J 1987, 6:1481-1491.

16. Bellomy GR, Mossing MC, Record MT: Physical properties of DNA in vivo as probed by the length pependence of the lac operator looping process. Biochemistry (Mosc) 1988, 27:3900-3906.

17. Oehler S, Eismann ER, Kramer H, Muller-Hill B: The three operators of the lac operon cooperate in repression. EMBO J 1990, 9:973-979.

18. Law SM, Bellomy GR, Schlax PJ, Record MT Jr: In vivo thermodynamic analysis of repression with and without looping in lac constructs: estimates of free and local lac repressor concentrations and of physical properties of a region of supercoiled plasmid DNA in vivo. $J \mathrm{Mol} B i o /$ 1993, 230:161-173

19. Garcia HG, Sanchez A, Boedicker JQ, Osborne M, Gelles J, Kondev J, Phillips R: Operator sequence alters gene expression independently of transcription factor occupancy in bacteria. Cell Reports 2012 , 2:150-161.

20. Bond LM, Peters JP, Becker NA, Kahn JD, Maher LJ: Gene repression by minimal lac loops in vivo. Nucleic Acids Res 2010, 38:8072-8082.

21. Lee DH, Schleif RF: In vivo DNA loops in araCBAD: size limits and helical repeat. Proc Natl Acad Sci USA 1989, 86:476-480.

22. Zhang Y, McEwen AE, Crothers DM, Levene SD: Analysis of In-Vivo LacR-Mediated Gene Repression Based on the Mechanics of DNA Looping. PLoS One 2006, 1:e136.

23. Müller J, Oehler $S$, Müller-Hill B: Repression of lac promoter as a function of distance, phase and quality of an auxiliary lac operator. J Mol Biol 1996, 257:21-29.

24. Brosius J, Erfle M, Storella J: Spacing of the-10 and-35 regions in the tac promoter-effect on its in vivo activity. J Biol Chem 1985, 260:3539-3541.

25. Huang HH, Camsund D, Lindblad P, Heidorn T: Design and characterization of molecular tools for a synthetic biology approach towards developing cyanobacterial biotechnology. Nucleic Acids Res 2010, 38:2577-2593.

26. Imamura S, Asayama M: Sigma factors for cyanobacterial transcription. Gene Regul Syst Biol 2009, 3:65-87.

27. Geerts D, Bovy A, De Vrieze G, Borrias M, Weisbeek P: Inducible expression of heterologous genes targeted to a chromosomal platform in the cyanobacterium Synechococcus sp. PCC 7942. Microbiology 1995, 141(Pt 4):831-841.

28. Guerrero F, Carbonell V, Cossu M, Correddu D, Jones PR: Ethylene synthesis and regulated expression of recombinant protein in Synechocystis $\mathrm{sp}$. PCC 6803. PLoS One 2012, 7:e50470.

29. de Boer HA, Comstock LJ, Vasser M: The tac promoter: a functional hybrid derived from the trp and lac promoters. Proc Natl Acad Sci USA 1983, $80: 21-25$.

30. Elhai J: Strong and regulated promoters in the cyanobacterium Anabaena PCC 7120. FEMS Microbiol Lett 1993, 114:179-184.

31. Schneider GJ, Haselkorn R: RNA-polymerase subunit homology among cyanobacteria, other eubacteria, and archaebacteria. J Bacterio/ 1988, 170:4136-4140.

32. Xie WQ, Jager K, Potts M: Cyanobacterial RNA-Polymerase Genes rpoc1 and rpoc2 Correspond to rpoc of Escherichia coli. J Bacteriol 1989, 171:1967-1973.

33. Asayama M: Regulatory system for light-responsive gene expression in photosynthesizing bacteria: cis-elements and trans-acting factors in transcription and post-transcription. Biosci Biotechnol Biochem 2006, 70:565-573

34. The iGEM registry of standard biological parts. http://parts.igem.org/.

35. Griese M, Lange C, Soppa J: Ploidy in cyanobacteria. FEMS Microbiol Lett 2011, 323:124-131
36. Lutz $\mathrm{R}$, Bujard $\mathrm{H}$ : Independent and tight regulation of transcriptional units in Escherichia coli via the LacR/O, the TetR/O and AraC/I-1-I-2 regulatory elements. Nucleic Acids Res 1997, 25:1203-1210.

37. Mitschke J, Georg J, Scholz I, Sharma CM, Dienst D, Bantscheff J, Voss B, Steglich C, Wilde A, Vogel J, Hess WR: An experimentally anchored map of transcriptional start sites in the model cyanobacterium Synechocystis $\mathrm{sp}$ PCC6803. Proc Natl Acad Sci U S A 2011, 108:2124-2129.

38. Kelly JR, Rubin AJ, Davis JH, Ajo-Franklin CM, Cumbers J, Czar MJ, De Mora K, Glieberman AL, Monie DD, Endy D: Measuring the activity of BioBrick promoters using an in vivo reference standard. $J$ Biol Eng 2009, 3:4.

39. Dong GG, Kim Yl, Golden SS: Simplicity and complexity in the cyanobacterial circadian clock mechanism. Curr Opin Genet Dev 2010, 20:619-625.

40. Temme K, Hill R, Segall-Shapiro TH, Moser F, Voigt CA: Modular control of multiple pathways using engineered orthogonal T7 polymerases. Nucleic Acids Res 2012, 40:8773-8781.

41. Shis DL, Bennett MR: Library of synthetic transcriptional AND gates built with split T7 RNA polymerase mutants. Proc Natl Acad Sci USA 2013, 110:5028-5033.

42. De Smit MH, Vanduin J: Secondary structure of the ribosome binding-site determines translational efficiency-a quantitative-analysis. Proc Natl Acad Sci USA 1990, 87:7668-7672.

43. Welch M, Villalobos A, Gustafsson C, Minshull J: You're one in a googol: optimizing genes for protein expression. J R Soc Interface 2009, 6(4):S467-S476.

44. Saecker RM, Record MT Jr, Dehaseth PL: Mechanism of bacterial transcription initiation: RNA polymerase-promoter binding, isomerization to initiation-competent open complexes, and initiation of RNA synthesis. J Mol Biol 2011, 412:754-771.

45. Sclavi B, Zaychikov E, Rogozina A, Walther F, Buckle M, Heumann H: Real-time characterization of intermediates in the pathway to open complex formation by Escherichia coli RNA polymerase at the T7A1 promoter. Proc Natl Acad Sci USA 2005, 102:4706-4711.

46. Chakraborty A, Wang D, Ebright YW, Korlann Y, Kortkhonjia E, Kim T, Chowdhury S, Wigneshweraraj S, Irschik H, Jansen R, et al: Opening and closing of the bacterial RNA polymerase clamp. Science 2012, 337:591-595.

47. Schyns G, Jia L, Coursin T, Tandeau de Marsac N, Houmard J: Promoter recognition by a cyanobacterial RNA polymerase: in vitro studies with the Calothrix sp. PCC 7601 transcriptional factors RcaA and RcaD. Plant Mol Biol 1998, 36:649-659.

48. Imashimizu M, Fujiwara S, Tanigawa R, Tanaka K, Hirokawa T, Nakajima Y, Higo J, Tsuzuki M: Thymine at-5 is crucial for cpc promoter activity of Synechocystis sp. strain PCC 6714. J Bacteriol 2003, 185:6477-6480.

49. Imashimizu M, Tanaka K, Shimamoto N: Comparative study of cyanobacterial and E. coli RNA Polymerases: misincorporation, abortive transcription, and dependence on divalent cations. Genet Res Int 2011:572689.

50. Leveau JH, Lindow SE: Predictive and interpretive simulation of green fluorescent protein expression in reporter bacteria. J Bacteriol 2001, 183:6752-6762.

51. Becker NA, Kahn JD, Maher $L J$ III: Bacterial repression loops require enhanced DNA flexibility. J Mol Biol 2005, 349:716-730.

52. Becker NA, Kahn JD, Maher $L J$ III: Eukaryotic HMGB proteins as replacements for $\mathrm{HU}$ in $E$. coli repression loop formation. Nucleic Acids Res 2008, 36:4009-4021.

53. CyanoBase. http://genome.microbedb.jp/CyanoBase.

54. Goodson KA, Wang Z, Haeusler AR, Kahn JD, English DS: Lacl-DNA-IPTG loops: equilibria among conformations by single-molecule FRET. J Phys Chem B 2013, 117:4713-4722.

55. Becker NA, Peters JP, Maher LJ III, Lionberger TA: Mechanism of promoter repression by Lac repressor-DNA loops. Nucleic Acids Res 2012, 41:156-166.

56. Mutalik VK, Guimaraes JC, Cambray G, Lam C, Christoffersen MJ, Mai QA, Tran AB, Paull M, Keasling JD, Arkin AP, Endy D: Precise and reliable gene expression via standard transcription and translation initiation elements. Nat Methods 2013, 10:353-354.

57. Müller-Hill B, Crapo L, Gilbert W: Mutants that make more lac repressor. Proc Natl Acad Sci USA 1968, 59:1259-1264. 
58. Stanier RY, Kunisawa R, Mandel M, Cohenbaz G: Purification and properties of unicellular blue-green algae (Order Chroococcales). Bacteriol Rev 1971, 35:171.

59. Shetty RP, Endy D, Knight TF Jr: Engineering BioBrick vectors from BioBrick parts. J Biol Eng 2008, 2:5.

60. Elhai J, Vepritskiy A, Muro-Pastor AM, Flores E, Wolk CP: Reduction of conjugal transfer efficiency by three restriction activities of Anabaena sp. strain PCC 7120. J Bacteriol 1997, 179:1998-2005.

61. Milkowski C, Quinones A, Hagemann M: A DNA fragment from the cyanobacterium Synechocystis sp. PCC 6803 mediates gene expression inducible by osmotic stress in E. coli. Curr Microbiol 1998, 37:108-116.

62. Kunert A, Hagemann M, Erdmann N: Construction of promoter probe vectors for Synechocystis sp. PCC 6803 using the light-emitting reporter systems Gfp and LuxAB. J Microbiol Methods 2000, 41:185-194.

63. Gibson DG, Young L, Chuang RY, Venter JC, Hutchison CA, Smith HO: Enzymatic assembly of DNA molecules up to several hundred kilobases. Nat Methods 2009, 6:U341-U343.

64. Hillson NJ, Rosengarten RD, Keasling JD: j5 DNA assembly design automation software. ACS Synthetic Biol 2012, 1:14-21.

65. Kremers GJ, Goedhart J, van den Heuvel DJ, Gerritsen HC, Gadella TWJ: Improved green and blue fluorescent proteins for expression in bacteria and mammalian cells. Biochemistry (Mosc) 2007, 46:3775-3783.

doi:10.1186/1754-1611-8-4

Cite this article as: Camsund et al:: Design and analysis of Lacl-repressed promoters and DNA-looping in a cyanobacterium. Journal of Biological Engineering 2014 8:4.

\section{Submit your next manuscript to BioMed Central and take full advantage of:}

- Convenient online submission

- Thorough peer review

- No space constraints or color figure charges

- Immediate publication on acceptance

- Inclusion in PubMed, CAS, Scopus and Google Scholar

- Research which is freely available for redistribution 